\title{
A scalar field inducing a non-metrical contribution to gravitational acceleration and a compatible add-on to light deflection
}

\section{Erhard Scholz ${ }^{1}$}

Received: 28 September 2019 / Accepted: 20 April 2020 / Published online: 7 May 2020 (c) The Author(s) 2020

\begin{abstract}
A scalar field model for explaining the anomalous acceleration and light deflection at galactic and cluster scales, without further dark matter, is presented. It is formulated in a scale covariant scalar tensor theory of gravity in the framework of integrable Weyl geometry and presupposes two different phases for the scalar field, like the superfluid approach of Berezhiani/Khoury. In low acceleration regimes of static gravitational fields (in the Einstein frame) with accordingly low values of the scalar field gradient, the scalar field Lagrangian combines a cubic kinetic term similar to the "a-quadratic" Lagrangian used in the first covariant generalization of MOND (RAQUAL) (Bekenstein and Milgrom in Astrophys J 286:7-14, 1984) and a second order derivative term introduced by Novello et al. in the context of a Weyl geometric approach to cosmology (Novello et al. in Int J Mod Phys D 1:641-677, 1993; de Oliveira et al. in Class Quantum Gravity 14(10):2833-2843, 1997). In varying with regard to $\phi$ the latter is variationally equivalent to a first order expression. The scalar field equation thus remains of order two. In the Einstein frame it assumes the form of a covariant generalization of the Milgrom equation known from the classical MOND approach in the deep MOND regime. It implies a corresponding "non-metrical" contribution to the acceleration of free fall trajectories. In contrast to pure RAQUAL, the second order derivative term of the Lagrangian leads to a non-negligible contribution to the energy momentum tensor and an add-on to the light deflection potential in beautiful agreement with the dynamics of low velocity trajectories. Although the model takes up important ingredients from the usual RAQUAL approach, it differs essentially from the latter.-In higher sectional curvature regions, respectively for higher accelerations in static fields, the scalar field Lagrangian consists of a Jordan-Brans-Dicke term with sufficiently high value of the JBD-constant to satisfy empirical constraints. Here the dynamics agrees effectively with the one of Einstein gravity.
\end{abstract}

Erhard Scholz

scholz@math.uni-wuppertal.de

1 Faculty of Mathematics/Natural Sciences, and Interdisciplinary Centre for History and Philosophy of Science, University of Wuppertal, Wuppertal, Germany 
Keywords Galaxy rotation curves - Relativistic MOND - Scalar field - Scale covariance · Integrable Weyl geometry · Light deflection · RAQUAL · Coma cluster

\section{Contents}

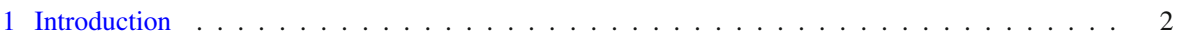

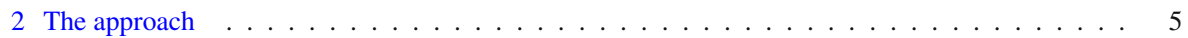

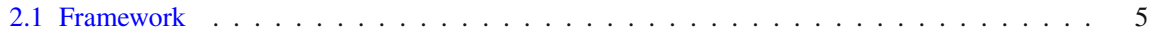

2.2 Lagrange density . . . . . . . . . . . . . . . . . . . . . . . . 6

2.3 Einstein equation and its energy momentum terms . . . . . . . . . . . . . . . 9

2.4 Scalar field equation $\ldots \ldots \ldots \ldots \ldots \ldots \ldots \ldots \ldots$

2.5 Free fall trajectories . . . . . . . . . . . . . . . . . . . . . . . 13

2.6 Einstein gauge and the measured values of observable quantities . . . . . . . . . . . . . . 14

3 MG regime and Newton-Milgrom approximation . . . . . . . . . . . . . . . . . . 16

3.1 The modification of Einstein gravity in the MG regime . . . . . . . . . . . . . . . . 16

3.2 Newton-Milgrom approximation . . . . . . . . . . . . . . . . . . . . 17

3.3 The scalar field energy-momentum in the Milgrom approximation . . . . . . . . . . . . . . 19

3.4 Light deflection due to the scalar field . . . . . . . . . . . . . . . . . . . . . . . . . . . . . . . . . . .

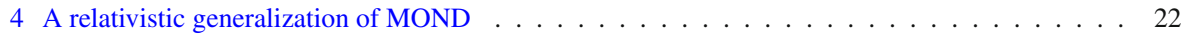

4.1 Comparison with MOND for simple systems . . . . . . . . . . . . . . . . . . . . 22

4.2 A short look at galactic dynamics . . . . . . . . . . . . . . . . . . . . . . . 24

4.3 From stars to galaxies and from galaxies to clusters . . . . . . . . . . . . . . . 25

4.4 A heuristic discussion of cluster dynamics . . . . . . . . . . . . . . . . . . . . 28

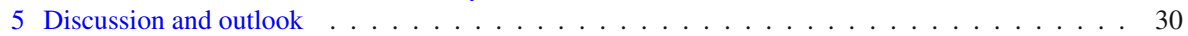

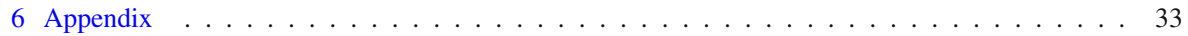

6.1 Weylian metric, derivative operators, curvatures, Einstein tensor . . . . . . . . . . 33

6.2 Some useful formulas . . . . . . . . . . . . . . . . . . . . . . . . . . . . . . . . . . . . . . . . . . .

6.3 Geroch-Jang theorem . . . . . . . . . . . . . . . . . . . 35

6.4 Short comment on gravitational light deflection . . . . . . . . . . . . . . . . . 36

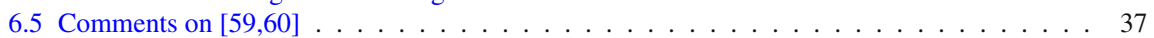

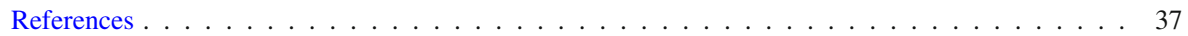

\section{Introduction}

A cubic term in the partial derivatives of a scalar field $\phi$ was introduced by Milgrom and Bekenstein in their Lagrangian formulation of Milgrom's "modified Newtonian dynamics" (MOND) and its first relativistic generalization RAQUAL (an acronym for a "relativistic a-quadratic Lagrangian") [3]. Similar cubic expressions in first derivatives of additional fields have become a generic feature of models attempting to explain the flat rotation curves of galaxies by a modification of gravity rather than by dark matter. The physical origin of these Lagrangians and the reason for the different types of dynamics in extremely weak gravitational regimes (MOND-like) and in regions with stronger gravitation (Newtonian or Einsteinian) have remained open. Khoury, Berezhiani et al. propose an interesting path for shedding light on both questions [4-7]. According to them, fractional powers of $(\partial \phi)^{2}$ are not uncommon in the effective Lagrangians of dynamical excitations in superfluids; moreover the formation of a superfluid phase of a (hypothetical) underlying substrate is dependent on special conditions which make it natural to assume different phases for the substrate (which they still leave open) in gravitationally strongly differing spacetime regions. They also 
try to connect their superfluid dark matter with modified gravity of a MONDian type (in their case TeVeS).

The authors of RAQUAL themselves saw and criticized the limitations of their approach. Although it leads to a MOND-like modification (of Newton/Einstein gravity) for low velocity orbits, it is unable to explain the observed additional gravitational lensing effects. Moreover it admits excitations with superluminal propagation. Various other relativistic generalizations of MOND, in particular TeVeS ("Tensor-vectorscalar") theory [2,64], "Einstein-aether" theories [34], have been proposed to cure these deficiencies. These models often introduce an additional vector field besides one or more scalar ones as part of the gravitational structure, and use strange geometries as their spacetime framework with, e.g., two different metrics with a non-conformal transformation between them (TeVeS), or a breaking of Lorentz invariance in the infinitesimal domain (Einstein ether). Motivation for some features of these generalizations came from cosmology rather than from (or in addition to) astrophysics [21, sec. 7], [14, sec. 3].

The present paper comes back to presupposing only one additional scalar field like RAQUAL, but with a different Lagrangian density and assuming two different phases for the scalar field, like in the superfluid approach of Berezhiani/Khoury. In the Einstein frame and very low acceleration regions of static gravitational fields (accordingly for very low values of the sectional curvature for the Einstein metric), the scalar field Lagrangian combines a cubic kinetic term $(\partial \phi)^{3}$, similar to RAQUAL in the deep MOND regime, with a second order derivative self-interaction term (type $\partial^{2} \phi$ ) first studied by Novello et al. in the context of a Weyl geometric approach to cosmology $[17,42]$, and a conformally coupled second order term. This phase will be called the $M G$ (modified gravity) regime of the present model. All terms of the Lagrangian are formulated in a locally scale covariant form with integrable Weyl geometry as the geometrical framework for the spacetime structure and scale covariant derivatives of the fields. In higher sectional curvature regions, respectively for higher accelerations in static fields, the scalar field Lagrangian consists of a Jordan-Brans-Dicke (JBD) term with sufficiently high value of the coefficient to satisfy empirical constraints which lead effectively to the dynamics of Einstein gravity. This will be called the $e E G$ (effective Einstein gravity) regime.

The scalar field, $\phi=C e^{-\sigma}$ in Riemann gauge (Jordan frame), plays a crucial role in modifying the spacetime geometry. Its logarithm $\sigma$ is the potential of an integrable Weylian scale connection $\varphi=-d \sigma$ in Einstein frame/gauge. In the eEG-regime its effects are negligible, while in the MG regime it modifies the free fall trajectories in Einstein gauge (Einstein frame) similar to RAQUAL. While varying with regard to $\phi$ the second order scale covariant derivative $D_{v} D^{v} \phi$ is variationally equivalent to a first order expression, because the second order partial derivatives $\partial_{\nu} \partial^{\nu} \phi$ occur in a divergence term involving the metric $g$. The scalar field equation thus remains of order two. This was already observed by the Brazilian group of physicists and used for constructing their cosmological model.

Due to the combined cubic and second order Lagrangian (and adding the conformally coupled second order term), the scalar field equation takes on the form of a covariant generalization of the Milgrom equation, if one goes over to the Einstein frame. Its flat (Euclidean) counter-part is known from the deep MOND regime in the 
classical MOND approach and serves as an approximation for regions in which the Riemannian curvature components are small enough for allowing a Newton approximation of the Einstein equation. Both together will be called a Newton-Milgrom approximation of the present model in the MG-regime. The solution of the Milgrom equation implies a corresponding non-metrical contribution to the acceleration of free fall trajectories.

In contrast to pure RAQUAL, the second order $\left(\partial^{2} \phi\right)$ term leads to a non-negligible contribution to the energy momentum tensor of the scalar field. It comes as a surprise that, although the scalar field energy momentum does not contribute to the source of the Newton approximation ( $\rho+\sum_{1}^{3} p_{j}$ of the scalar field vanishes), it does so for the calculation of the gravitational light deflection in the MG-regime. In the central symmetric case it leads to an add-on of the light deflection potential in beautiful agreement with the dynamics of low velocity trajectories. We may conjecture that this also holds generally.

Finally the scalar field energy seems to play an important role for the modification of gravity also in hierarchical structured systems. In very weak acceleration configurations the solutions of the Milgrom approximations at different levels, e.g., galactic and cluster, may (tentatively) be superimposed linearly. For clusters this leads to an estimate of the overall modification of gravity different from other MOND-like theories, measurable in a larger "total mass" (only partially phantom) attributed to the gravitational effects of the scalar field via its influence on the non-metrical component of the affine connection. The energy-momentum of the scalar field can be considered as the expression of a kind of "dark matter" sui generis with repercussion in particular for cluster dynamics. All in all the present model differs considerably from RAQUAL although it takes up important ingredients from the the latter.

The organization of the paper is as follows: The basic features of the present approach are presented in the first section. Section 2.1, supported by Appendix 6.1, gives a refreshment of the geometrical framework of integrable Weyl geometry and introduces the respective notations. In a scale gauge (roughly corresponding the choice of a frame in Jordan-Brans-Dicke theory) the complete data of a Weylian metric are given in the integrable case by a pair $(g, \varphi)$ consisting of a (pseudo-) Riemannian metric $g$ and a closed differential 1-form, or in physics terminology a pure gauge co-vector field $\varphi$. Section 2.2 formulates the scale invariant Lagrangian densities of the model, in particular those for the two different phases of the scalar field. The derivation of the modified (Weyl geometric) Einstein equation and the scalar field equation for both regimes follows in Sects. 2.3 and 2.4. In the first (eEG) regime the dynamics is like in JBD theory; we therefore have to assume a sufficiently high value for the coupling coefficient of the JBD kinetic term in order to arrive effectively at the dynamics of Einstein gravity. In the second (MG) regime we derive a covariant generalization, Eq. (29), of the non-linear Poisson equation known from classical MOND kinematics. By obvious reasons it will be called the covariant Milgrom equation. Free fall trajectories in the present (integrable Weyl geometric) framework are studied in Sect. 2.5. In the Einstein gauge (comparable to the Einstein frame of JBD theory) an additional term of the acceleration of test bodies arises. It is due to the scale connection determined by the scalar field, Eq. (34). Finally a discussion of the question in which scale measurable quantities are most directly expressed leads us again to the Einstein gauge (Sect. 2.6). 
The second section deals with the dynamics in the MG regime. Section 3.1 analyses the modification due to the scalar field of the Einstein equation for the Riemannian part $g$ of the Weylian metric. In Sect. 3.2 we turn towards the quasi-static weak field approximations for the Einstein equation and the Milgrom equation. The first one boils down to a Newton approximation like in Einstein gravity. The second one has the form of the non-linear Poisson equation of deep MOND. Both together constitute the NewtonMilgrom approximation of our approach. The solution of the Milgrom approximation leads to an additional acceleration of a form known from the deep MOND regime in the usual MOND approach. The energy momentum tensor of the scalar field in the Milgrom approximation has intriguing properties (Sect.3.3f). Although at a first glance its energy density might seem consistent with the additional acceleration (it agrees with the phantom energy density associated to the additional acceleration of the scale connection), it does not contribute to the source term of the Newton approximation because of its negative pressure terms. On the other hand, it does contribute to light deflection. An investigation of the spatial components of Riemannian component of the metric in the weak field approximation and Einstein gauge for the central symmetric case leads to a light deflection potential consistent with the additional acceleration of the scale connection (Sect. 3.4).

This is a peculiar result for the present model, up to now unexpected for relativistic generalizations of MOND with only one additional scalar field. The respective MONDtypical interpolation functions and their feasibility for galactic dynamics are discussed in Sects. 4.1 and 4.2. It also may have important repercussions for the dynamics of galaxy clusters (Sects. 4.3, 4.4). Additional questions not dealt with in detail in this paper are shortly discussed in the final Sect. 5.

\section{The approach}

\subsection{Framework}

We use a scale covariant generalization of Einstein gravity formulated in the framework of integrable Weyl geometry with one additional degree of freedom only, incorporated by a gravitationally coupled scalar field (see Appendix 6.1). The Weylian metric is given in any (scale) gauge by the data $(g, \varphi)$, where $g=g_{\mu \nu} d x^{\mu} d x^{\nu}$ is the Riemannian component of the (Weylian) metric, here of Lorentzian signature type $(-+++)$, and $\varphi$ represents an integrable Weylian scale connection, given by a closed differential 1 -form (a pure gauge co-vector field) $\varphi=\varphi_{\nu} d x^{\nu}$ satisfying $\partial_{\mu} \varphi_{\nu}-\partial_{\nu} \varphi_{\mu}=0$. In addition we assume a real valued, positive scalar field $\phi$, in Riemann gauge (Jordan frame) given by $\phi=C e^{-\sigma}$ and scaling with Weyl weight -1 . It plays a part in the gravitational structure of our model.

Being pure gauge, $\varphi$ can be integrated away locally; then the Weylian metric acquires the form $(\tilde{g}, 0)$ and reduces to the (peudo-) Riemannian metric $\tilde{g}$. By obvious reasons this scale choice will be called the Riemann gauge of the Weylian metric. 
It corresponds to the Jordan frame of Jordan-Brans-Dicke (JBD) theory. ${ }^{1}$ Here the scalar field can be written as

$$
\tilde{\phi}=\phi_{0} e^{-\sigma} \text {. }
$$

$\sigma$ is scale invariant by definition. Below we see that it plays the role of a potential for modifications of the kinematics of free fall in Einstein gauge (34). We thus call it the invariant potential of the scale connection. The scale connection does not entail a dynamical degree of freedom of its own; it arises from rescaling, $\varphi_{\nu}=\partial_{\nu} \omega$ (see Eq. 2). In Einstein gauge/frame, in which the scalar field is scaled to a constant value, it expresses the dynamical content of the scalar field in the sense of $\varphi_{\nu}=\partial_{\nu} \sigma$.

The data in any other scale gauge arise from the Riemann gauge by (length-) rescaling with a real valued function $\Omega=e^{\omega}$ :

$$
g \doteq e^{2 \omega} g, \quad \varphi_{\nu} \doteq-\partial_{\nu} \omega \quad(\varphi \doteq-d \omega), \quad \phi \doteq \phi_{0} e^{-(\sigma+\omega)}
$$

Here $\doteq$ denotes equalities which are scale dependent. The Einstein gauge (also Einstein frame, but see fn. 1 with metrical data $(\hat{g}, \hat{\varphi})$ and scalar field $\hat{\phi}$ is specified by the condition that the scalar field is scaled to a constant, $\hat{\phi} \doteq \phi_{0}$, thus:

$$
\hat{g} \underset{\overline{E g}}{\doteq} e^{-2 \sigma} g \quad \hat{\varphi}_{\nu} \underset{\overline{E g}}{\doteq} \partial_{\nu} \sigma \quad \hat{\phi} \underset{\overline{E g}}{\doteq} \phi_{0} \quad \text { constant }
$$

where $\underset{\overline{E g}}{\doteq}$ denotes equality in Einstein gauge; similarly $\underset{\overline{R g}}{\doteq}$ for Riemann gauge.

Partial derivatives are denoted as usual by $\partial$. For covariant derivations we have to distinguish between the Levi-Civita derivative ${ }_{g} \nabla$ with regard to the Riemannian component $g$ of the Weylian metric, the scale invariant covariant derivative $\nabla$ with regard to the Weylian metric given by $(g, \varphi)$, and the scale covariant derivative $D$ of fields (which themselves are scale dependent). For technical details see Appendix 6.1.

It is convenient to introduce a sign symbol $\epsilon_{X}$ for scalar fields $X$, depending on the signature type of the scale covariant gradient $D X$ :

$$
\epsilon_{X}=\left\{\begin{array}{cl}
+1 & \text { for } D X \text { spacelike } \\
-1 & \text { for } D X \text { timelike or null }
\end{array}\right.
$$

Then the norm of a scale covariant gradient is $|D X|=\left(\epsilon_{X} D_{\nu} X D^{v} X\right)^{\frac{1}{2}}$ and in particular $|D \phi| \underset{\overline{E g}}{\doteq} \phi_{0}|\nabla \sigma|$.

\subsection{Lagrange density}

We assume a scale invariant Lagrangian density of the form

$$
\mathfrak{L}=L \sqrt{|\operatorname{det} g|}, \quad L=L_{H}+L_{D \phi}+L_{V}+L_{b a r},
$$

\footnotetext{
${ }^{1}$ The usual terminology of "frame" is ambiguous. Often it is used in the sense of choosing an orthonormal frame (tetrad); in JBD theory it indicates only a choice of scale, leaving coordinate choice and tetrad choice (if any) open.
} 
with a gravitational term $L_{H}$, the kinetic and potential terms of the scalar field $L_{D \phi}, L_{V(\phi)}$, and a matter term $L_{b a r}$ which we do not specify here. It serves as a placeholder for the classical action of baryonic matter. In order to cancel the scale weight of the volume element $\sqrt{|\operatorname{det} g|}$, all contributions $L_{X}$ have to be given in scale covariant form of Weyl weight $w\left(L_{X}\right)=-n=-4$. For $L_{m}$ one has to introduce appropriate scaling conventions for its constituent fields without assuming a direct coupling to $\phi$.

The gravitational action is similar to JBD-theory (in Riemann gauge the two are even equal):

$$
L_{H}=\frac{1}{2}(\xi \phi)^{2} R, \quad \text { with } \xi=\frac{E_{P}}{\phi_{0}},
$$

where $R$ denotes the Weyl geometric scalar curvature (scale covariant of weight $w(R)=-2$, see Appendices 6.1, 6.2). $\xi$ is a hierarchy factor between the Planck scale energy $E_{P}$ and the energy level of the scalar field, indicated by $\phi_{0}$.

The scale weight condition

$$
L_{V}=-V \text { with } w(V)=-4
$$

constrains the form of $V$ to a quartic monomial in the scalar field,

$$
V=V(\phi)=\frac{\lambda_{4}}{4} \phi^{4}
$$

or to a biquadratic coupling of $\phi$ to the norm $h$ of the Higgs field $\left(h^{2}=\Phi^{\dagger} \Phi\right)$, with or without separate quartic term for $\phi:^{2}$

$$
V(\phi, h)=\frac{\lambda}{4}\left(\left(h^{2}-(\eta \phi)^{2}\right)\right)^{2}\left[+\frac{\lambda_{4}}{4} \phi^{4}\right]
$$

$\eta=\frac{v}{\phi_{0}}$ is a new hierarchy factor between the electroweak energy level $v \approx 246 \mathrm{GeV}$ and the energy of the scalar field $\phi$. With (8) the gravitational scalar field $\phi$ is able to enter the Higgs portal in a moderate form (via the potential term only). In the case $\eta \sim 1$ one may expect $\lambda_{4}=0 .^{3}$

The kinetic term $L_{D \phi}$ of the model superimposes three terms:

$$
L_{D \phi}=L_{D \phi^{2}}+L_{D \phi^{3}}+L_{D^{2} \phi}
$$

where $L_{D \phi^{2}}$, see (10), denotes the usual quadratic kinetic term with coefficient $\alpha$ similar to JBD written in scale covariant form, $L_{D \phi^{3}}(11,12)$ with coefficient $\beta$ is the cubic term adapted from the RAQUAL Lagrangian [3], and $L_{D^{2} \phi}$ (13) with coefficient $\gamma$ the second order term from the Brazilian approach to gravity mentioned

\footnotetext{
${ }^{2}$ Similar to [63].

${ }^{3}$ In this case a hierarchy factor $\xi^{\prime}$ (different from $\xi$ ) between the MONDian constant $a_{0}$ and $\phi_{0}$ has to be introduced in the $L_{D \phi^{3}}$ Eq. (11).
} 
above $[17,42] .{ }^{4}$ All terms are rewritten in scale covariant form (weight -4) with scale covariant derivatives $D$ in a general scale gauge and in Einstein gauge:

$$
\begin{aligned}
& L_{D \phi^{2}}=-\frac{\alpha}{2} D_{\nu}(\xi \phi) D^{\nu}(\xi \phi)=-\epsilon_{\phi} \frac{\alpha}{2}|D(\xi \phi)|^{2} \\
& \underset{\overline{E g}}{\doteq}-\frac{\alpha}{2}\left(\xi \phi_{0}\right)^{2} \partial_{\lambda} \sigma \partial^{\lambda} \sigma \\
& L_{D \phi^{3}}=-\epsilon_{\phi} \frac{2}{3} \beta \phi^{-2}\left[\epsilon_{\phi} D_{\nu}(\xi \phi) D^{\nu}(\xi \phi)\right]^{\frac{3}{2}}=-\epsilon_{\phi} \frac{2}{3} \beta \phi^{-2}|D(\xi \phi)|^{3} \\
& \underset{\overline{E g}}{\doteq}-\epsilon_{\sigma} \frac{2}{3} \beta\left(\xi \phi_{0}\right)^{2}\left(\xi^{-1} \phi_{0}\right)^{-1}|\nabla \sigma|^{3} \\
& L_{D^{2} \phi}=\frac{\gamma}{2}(\xi \phi) D_{\nu} D^{v}(\xi \phi)=\frac{\gamma}{2} \xi^{2} \phi D_{\nu} D^{v} \phi \\
& \underset{\overline{E g}}{\doteq}-\frac{\gamma}{2}\left(\xi \phi_{0}\right)^{2}\left(\nabla_{\nu} \partial^{v} \sigma-3 \partial_{\nu} \partial^{v} \sigma\right) \\
& \underset{\overline{E g}}{\doteq}-\frac{\gamma}{2}\left(\xi \phi_{0}\right)^{2}\left({ }_{g} \nabla_{\nu} \partial^{v} \sigma+\partial_{\nu} \partial^{v} \sigma\right)
\end{aligned}
$$

We basically assume two regimes in which the scalar field is governed by different Lagrangians, the $e E G$ regime (effectively Einstein gravity) in which the scalar field underlies a JBD Lagrangian with a large coefficient $\alpha \geq 10^{5}$ which leads effectively to the dynamics of Einstein gravity, and the $M G$ (modified gravity) regime with a MOND-like dynamics. The Lagrangian of the MG regime is "switched on" under specific conditions for the scalar curvature; or the other way round, the MG regime is switched off if the gradient of the scalar field surpasses a critical value roughly an order of magnitude below the MOND constant $a_{0}$. A physical explanation of this behaviour of the present Larangian may result from a superfluid hypothesis of an Einstein-Bose condensate similar to theory of Khoury and Berezhiani [4,6,7]. According to these authors the superfluid phase is suppressed for large phonon gradients of the superfluid which leads to a gradual transition to Newton/Einstein gravity with an increasingly smaller amount of superfluid phase of the condensate $[5, \S 5] .^{5}$

$$
L_{D \phi}=L_{D \phi}(\alpha, \beta, \gamma) \text { with } \begin{cases}\alpha \gg 1, \beta=\gamma=0 & \text { in the eEG regime } \\ \alpha=-6, \beta, \gamma \neq 0 & \text { in the MG regime }\end{cases}
$$

In the sequel we find a preference for setting $\beta=6$ (see (38)) and reasons for assuming $\gamma=4$ (Result b) on p. 20). So we can put the Lagrangians

$$
L_{e E G}=L_{D \phi}\left(\alpha_{1}, 0,0\right), \quad L_{M G}=L_{D_{\phi}}(-6,6,4)
$$

(with some $\alpha_{1} \gg 1$ ) for the effective Einstein and the modified gravity regimes respectively.

\footnotetext{
${ }^{4}$ The authors of $[17,42]$ assume a breaking of scale symmetry to the Einstein gauge, which is here avoided at the level of the general Lagrangian.

${ }^{5}$ Berezhiani/Khoury assume also a fading out of the superfluid phase for low pressures, i.e. far beyond galaxies, leading to a phase with usual dark matter already at the cluster level. This is not the case for the present approach, but may be considered at the level of voids.
} 
At the moment there are no reasons for assuming a specific law for the intermediate (im) region between the domains of eEG and MG. One may like, however, to postulate a transition function $\chi_{i m}$ with $\chi_{i m}(x)=1$ in the MG region, $\chi_{i m}(x)=0$ in the eEG region and a smooth transition, such that in the intermediate region

$$
L_{i m}=\left(1-\chi_{i m}\right) L_{e E G}+\chi_{i m} L_{M G}
$$

This is at least a formal device for shunting out our ignorance (even at the phenomenological level) of the physics in the intermediate region.

In the eEG regime we thus have $L_{D \phi}=L_{D \phi^{2}}$ with $\alpha>10^{5}$ which makes it compatible with Einstein gravity in the solar system [75]; in the MG regime all three contributions to $L_{D \phi}$ are switched on. In the MG regime a fractional power $\frac{3}{2}$ of the quadratic term is turned on. According to Khoury/Berezhiani that is similar to what can be expected in the superfluid phase of a bosonic condensate [7]. Fractional powers (with exponent $\frac{3}{2}$ ) of quadratic kinetic terms are typical for the known Lagrangian field theories with MOND-like phenomenology (among them in particular the original RAQUAL approach [3], TeVeS [2], the superfluid theory of Khoury/Berezhiani [6] and Hossenfelder's covariant version of "emergent" gravity [30]). $L_{D^{2} \phi}$ contributes considerably to the energy-momentum of the scalar field and leads to an important difference to the original RAQUAL approach. According to our hypothesis (14) is switched on in the MG regime together with the cubic kinetic term, while the coefficient of the ordinary quadratic term is shifted to conformal coupling, i.e. to a gravitationally inert state.

At the end of Sect. 3.2 we come back to distinguishing criteria between the different regimes in the context of a weak field (Newton-Milgrom) approximation. The criteria given there are based on rough estimates on the validity region for a MONDlike dynamics. A theoretical underpinning for such a separation of regions is still conjectural, but there are arguments for the appearance of "kinematic screening" of scalar fields in the theory of superfluids. ${ }^{6}$ A better consolidated theory would probably formulate the distinguishing criteria for the two regimes in terms of the scalar field gradient.

\subsection{Einstein equation and its energy momentum terms}

In order to take full advantage of the scaling symmetry of the Lagrangian, the variational derivatives are dealt with in a scale covariant framework like in other gauge symmetric theories [23, p. 524ff.]. The scale covariant Euler-Lagrange equation with regard to $g$,

$$
\frac{\partial \mathfrak{L}}{\partial g^{\mu \nu}}-D_{\lambda} \frac{\partial \mathfrak{L}}{\partial\left(D_{\lambda} g^{\mu \nu}\right)}
$$

\footnotetext{
${ }^{6}$ Berezhiani/Khoury argue in [4, p. 4]: "The EFE (external field effect, ES) is an example of a more general phenomenon in scalar field theories known as kinetic screening [...]. In theories with gradient interactions, non-linearities in the scalar field gradient-the scalar acceleration-can result in the suppression of the scalar field effects and the local recovery of standard gravity. See [75] for a review." ([75] refers to our [35].)
} 
can be calculated in any scale gauge if in the end the result is rewritten in scale covariant form. In our case, the integrable Weyl geometric context makes the calculation easy. In most cases, one can go to Riemann gauge and use the fact that all derivative operators

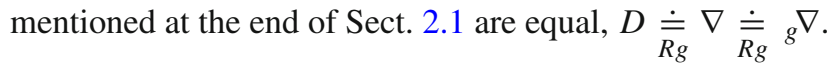

After multiplying the result with $(\xi \phi)^{-2}$ we arrive at the following scale invariant Einstein equation:

$$
G=R i c-\frac{R}{2} g=(\xi \phi)^{-2} T^{(b a r)}+\Theta^{(H)}+\sum_{X} \Theta^{(X)}
$$

Here Ric $=\left(R_{\mu \nu}\right), R$ and $G=\left(G_{\mu \nu}\right)$ denote the Weyl geometric Ricci tensor, scalar curvature, and Einstein tensor respectively (see Appendix 6.1). Ric is scale invariant by definition, $w(R)=-2, w(g)=2$. Therefore also the Weyl geometric Einstein tensor is scale invariant. Similar weight counts hold for all terms on the right hand side (r.h.s.) of Eq. (17). This equation also holds without assuming integrability of the Weylian scale connection $[9,19,65])$.

The Weyl geometric Einstein tensor in scale gauge $(g, \varphi)$ is the sum of a term ${ }_{g} G$ due to the Riemannian part of the metric $g$ and well known from Einstein gravity, and an expression ${ }_{\varphi} G$ containing the contributions of the scale connection (Appendix 6.1),

$$
G={ }_{g} G+{ }_{\varphi} G
$$

The energy momentum tensor of classical matter

$$
T_{\mu \nu}^{(\text {bar })}=-\frac{2}{\sqrt{|g|}} \frac{\delta \mathfrak{L}_{\text {bar }}}{\delta g^{\mu \nu}}
$$

calculated according to (16) is scale covariant with weight -2 which cancels against $w\left(\phi^{-2}\right)$. Similarly the $\Theta^{(X)}$ denote the scale invariant contributions to the energy momentum tensor of the scalar field, up to coefficient:

$$
\Theta^{(X)}=-(\xi \phi)^{-2} \frac{2}{\sqrt{|g|}} \frac{\delta \mathfrak{L}_{X}}{\delta g^{\mu \nu}}=-(\xi \phi)^{-2}\left(2 \frac{\partial L_{X}}{\partial g^{\mu \nu}}-L_{X} g_{\mu \nu}\right)
$$

Here $X$ is used as a dummy index for the constituents of the scalar field with the appropriate summation domains, i.e. $X \in\left\{D \phi^{2}, V\right\}$ in the eEG region, and $X \in$ $\left\{D \phi^{2}, D \phi^{3}, D^{2} \phi V\right\}$ in the MG regime with $\alpha=-6$.

$\Theta^{(H)}$ is the variational contribution of $\phi$ due to the non-minimal coupling in $\mathfrak{L}_{H}$. It is well known also in JBD theory $[11,24]$. Written in terms of the scale covariant differentiation operator $D$ of Weyl geometry it is [19,67]:

$$
\begin{aligned}
\Theta_{\mu \nu}^{(H)} & =\phi^{-2}\left(D_{(\mu} D_{\nu)} \phi^{2}-D_{\lambda} D^{\lambda} \phi^{2} g_{\mu \nu}\right) \\
& =2 \phi^{-2}\left(D_{\mu} \phi D_{\nu} \phi+\phi D_{(\mu} D_{\nu)} \phi-\left(\phi D_{\lambda} D^{\lambda} \phi+D_{\lambda} D^{\lambda} \phi\right) g_{\mu \nu}\right)
\end{aligned}
$$

Using the calculation in Appendix 6.2 this is in Einstein gauge

$$
\underset{\overline{\overline{E g}}}{-2}{ }_{g} \nabla_{(\mu} \partial_{\nu)} \sigma+8 \partial_{\mu} \sigma \partial_{\nu} \sigma-2\left({ }_{g} \square \sigma+\partial_{\lambda} \sigma \partial^{\lambda} \sigma\right)
$$


Here

$$
{ }_{g} \square=-{ }_{g} \nabla_{\lambda} \partial^{\lambda}
$$

denotes the d'Alembert operator with regard to the Levi-Civita connection $g_{g} \nabla$.

The interpretation of $\Theta^{(H)}$ in the literature varies; some authors consider it as a geometrico-gravitational contribution to the Einstein equation and put it on its left hand side (1.h.s.), others see it as part of the energy-momentum of the scalar field. ${ }^{7}$ In Sect. 4.1 we come back to it in our context.

The other energy expressions (most of them in scale invariant form) are

$$
\begin{aligned}
\Theta_{\mu \nu}^{\left(D \phi^{2}\right)}= & (\xi \phi)^{-2}\left(\alpha \xi^{2} D_{\mu} \phi D_{\nu} \phi+L_{D \phi^{2}} g_{\mu \nu}\right) \\
= & \alpha\left(\partial_{\mu} \sigma \partial_{\nu} \sigma-\frac{1}{2} \partial_{\lambda} \partial^{\lambda} \sigma g_{\mu \nu}\right) \\
\Theta_{\mu \nu}^{\left(D \phi^{3}\right)}= & 2 \beta \xi \phi^{-4}|D \phi| D_{\mu} \phi D_{\nu} \phi+(\xi \phi)^{-2} L_{D \phi^{3}} g_{\mu \nu} \\
& \doteq \overline{\overline{E g}}^{-1} \beta\left(\xi^{-1} \phi_{0}\right)^{-1}\left(|\nabla \sigma| \partial_{\mu} \sigma \partial_{\nu} \sigma-\frac{\epsilon_{\sigma}}{3}|\nabla \sigma|^{3} g_{\mu \nu}\right) \\
\Theta_{\mu \nu}^{\left(D^{2} \phi\right)}= & \gamma \phi^{-1}\left(-D_{\mu} D_{\nu} \phi+\frac{1}{2} D_{\lambda} D^{\lambda} \phi g_{\mu \nu}\right) \\
& \doteq{ }_{\overline{E g}}-\gamma\left({ }_{g} \nabla_{\mu} \partial_{\nu} \sigma-3 \partial_{\mu} \sigma \partial_{\nu} \sigma\right)+\frac{\gamma}{2}\left({ }_{g} \nabla_{\lambda} \sigma^{\lambda}-3 \partial_{\lambda} \sigma \partial^{\lambda} \sigma\right) g_{\mu \nu} \\
\Theta_{\mu \nu}^{(V)}= & (\xi \phi)^{-2} L_{V} g_{\mu \nu}
\end{aligned}
$$

Tracing the Einstein equation and multiplying it with $-(\xi \phi)^{2}$ leads to

$$
-2 L_{H}-\operatorname{tr} T^{(b a r)}+(\gamma+6) \xi^{2} \phi D_{\lambda} D^{\lambda} \phi+(\alpha+6) \xi^{2} D_{\lambda} \phi D^{\lambda} \phi-L_{D \phi^{3}}-4 L_{V}=0 .
$$

Here all possible contributions of the scalar field are included. In the JBD domain one has to set $\gamma=0$ and $\beta=0$, in the MG regime $\alpha=-6$.

\subsection{Scalar field equation}

The scale covariant variation with regard to $\phi, \frac{\delta L}{\delta \phi}=\frac{\partial L}{\partial \phi}-D_{\lambda} \frac{\partial L}{\partial\left(D_{\lambda} \phi\right)}$, contains the partial contributions (see Appendix 6.2 (87)):

$$
\begin{aligned}
& \frac{\delta L_{D \phi^{2}}}{\delta \phi}=\alpha \xi^{2} D_{\lambda} D^{\lambda} \phi \\
& \frac{\delta L_{D \phi^{3}}}{\delta \phi}=2 \beta \xi^{3} \phi^{-2} D_{\lambda}\left(|D \phi| D^{\lambda} \phi\right)+4 \phi^{-1} L_{D \phi^{3}}
\end{aligned}
$$

In the second line we encounter a scale covariant form of the non-linear modification of the d'Alembert operator typical for relativistic MOND theories.

\footnotetext{
7 This was done in [60].
} 
For $L_{D^{2} \phi}$ it is recommendable to consider the Einstein gauge (13). Because of

$$
{ }_{g} \nabla_{\lambda} \partial^{\lambda} \sigma=\frac{1}{\sqrt{|g|}} \partial_{\lambda}\left(\sqrt{|g|} \partial^{\lambda} \sigma\right)
$$

the second order derivative term of $\mathfrak{L}_{D^{2} \phi}$ in Einstein gauge is a divergence expression

$$
-\frac{\gamma}{2}\left(\xi \phi_{0}\right)^{2} \partial_{\lambda}\left(\sqrt{|g|} \partial^{\lambda} \sigma\right)
$$

For the variation of $\phi$ (with fixed $g$ ) its integral can be shifted to a boundary term outside the support of $\delta \phi$ and does not contribute to the Euler-Lagrange equation of the scalar field. ${ }^{8}$ The same does not hold while varying $g$. For the variation $\delta \phi$ in Einstein gauge only the term $-\frac{\gamma}{2}\left(\xi \phi_{0}\right) \partial_{\lambda} \sigma \partial^{\lambda} \sigma$ of (13) remains and leads to a second degree dynamcial equation for $\phi$, respectively $\sigma$. Its scale covariant form is

$$
L_{D^{2} \text { preduced }}=-\frac{\gamma}{2}\left(\xi \phi_{0}\right)^{2} D_{\lambda} \sigma D^{\lambda} \sigma
$$

it is of the same form as $L_{D \phi^{2}}(10)$. In terms of $\phi$ we find

$$
\frac{\delta L_{D^{2} \phi}}{\delta \phi}=\gamma \xi^{2} D_{\lambda} D^{\lambda} \phi .
$$

$L_{H}$ and $L_{V}$ are monomials in $\phi$ with $\frac{\delta \phi^{k}}{\delta \phi}=\frac{\partial \phi^{k}}{\partial \phi}=k \phi^{k-1}$.

After summing up and multiplying with $\phi$ we arrive at the gross scalar field equation covering both regimes of (14):

$$
2 L_{H}+(\alpha-\gamma) \xi^{2} \phi D_{\lambda} D^{\lambda} \phi+4 L_{D \phi^{3}}+2 \beta \xi^{3} \phi^{-1} D_{\lambda}\left(|D \phi| D^{\lambda} \phi\right)+4 L_{V}=0
$$

Addition of the traced Einstein equation (25) leads to the scale covariant, (net) scalar field equation. In arbitrary scale gauge it is:

$$
2 \beta \xi^{3} \phi^{-1} D_{\lambda}\left(|D \phi| D^{\lambda} \phi\right)+(\alpha+6) \xi^{2} D_{\lambda} \phi D^{\lambda} \phi+(\alpha+6) \xi^{2} \phi D_{\lambda} D^{\lambda} \phi+3 L_{D \phi^{3}}-\operatorname{tr} T^{(b a r)}=0
$$

In the $M G$ regime $(\alpha=-6)$ this boils down to

$$
2 \beta \xi^{3} \phi^{-1} D_{\lambda}\left(|D \phi| D^{\lambda} \phi\right)+3 L_{D \phi^{3}}=\operatorname{tr} T^{(\text {bar })} .
$$

Taking account of $D_{\lambda}\left(|D \phi| D^{\lambda} \phi\right) \underset{\overline{E g}}{\doteq}-\phi_{0}^{2} D_{\lambda}\left(|\nabla \sigma| \partial^{\lambda} \sigma\right), \phi \underset{\overline{E g}}{\doteq} \phi_{0}$, and for a perfect fluid energy tensor with energy density $\rho_{b a r}$ and pressure $p_{b a r}$ this becomes in Einstein gauge:

$$
D_{\lambda}\left(|\nabla \sigma| \partial^{\lambda} \sigma\right)-\epsilon_{\sigma}|\nabla \sigma|^{3} \underset{\overline{E g}}{\doteq} \frac{1}{2}\left(\xi \phi_{0}\right)^{-2}\left((\beta \xi)^{-1} \phi_{0}\right)\left(\rho_{b a r}-3 p_{b a r}\right)
$$

\footnotetext{
8 This has been noted by the authors of [42].
} 
With Eq. (86) of the Appendix,

$$
D_{\lambda}\left(|\nabla \sigma| \partial^{\lambda} \sigma\right) \underset{\overline{E g}}{\doteq} \nabla_{\lambda}\left(|\nabla \sigma| \partial^{\lambda} \sigma\right)+|\nabla \sigma| \partial_{\lambda} \sigma \partial^{\lambda} \sigma
$$

the cubic terms $|\nabla \sigma|^{3}$ cancel, and we arrive at the scalar field equation in the MG regime:

$$
{ }_{g} \nabla_{\lambda}\left(|\nabla \sigma| \partial^{\lambda} \sigma\right) \underset{\overline{E g}}{\doteq} \frac{1}{2}\left(\xi \phi_{0}\right)^{-2}\left((\beta \xi)^{-1} \phi_{0}\right) \quad(\rho-3 p)_{b a r}
$$

Let us define the covariant Milgrom operator ${ }_{g} \Delta_{M}$ in Einstein gauge, for any scalar field $X$ as

$$
{ }_{g} \Delta_{M} X: \underset{\overline{E g}}{\doteq} \nabla_{\lambda}\left(|\nabla X| \partial^{\lambda} X\right)
$$

For the flat metric and static fields $X$ this is the non-linear Laplace operator of the classical MOND theory $\Delta_{M} X=\nabla_{j}\left(|\nabla X| \partial^{j} X\right)$ (with the Euclidean $\nabla$ operator). We therefore call (29) the covariant Milgrom equation.

In the $e E G$ regime $(\beta=\gamma=0)$, on the other hand, we get

$$
(\alpha+6) \xi^{2}\left(D_{\lambda} \phi D^{\lambda} \phi+\phi D_{\lambda} D^{\lambda} \phi\right)=\operatorname{tr} T^{(b a r)},
$$

or

$$
D_{\lambda} D^{\lambda} \phi^{2}=\frac{2}{\alpha+6} \xi^{-2} \operatorname{tr} T^{(\text {bar })} .
$$

In Einstein gauge and for fluid matter this is

$$
g \square \sigma \underset{\overline{E g}}{\doteq} \frac{2\left(\xi \phi_{0}\right)^{-2}}{\alpha+6}(\rho-3 p),
$$

the scalar field equation of JBD theory in the Einstein frame (cf. [24, pp. 42, 72]). (Remember that $g \square$ denotes the covariant d'Alembert operator (20).)

\subsection{Free fall trajectories}

If we model the trajectories of test bodies by energy-momentum concentrated in arbitray small neighbourhoods of a timelike curve, like in the Geroch-Jang approach to the geodesic theorem for Einstein gravity [26], it can be shown that in integrable Weyl geometry (IWG) test bodies move along timelike geodesics like in Einstein gravity. One basically passes to the Riemann gauge and applies the "classical" Geroch-Jang theorem (Appendix 6.3).

The scale invariant geodesics $\tilde{\gamma}(\tau)$ of IWG,

$$
\frac{d}{d \tau} \dot{\tilde{\gamma}}^{\mu}+\Gamma_{\nu \lambda}^{\mu} \dot{\tilde{\gamma}}^{\nu} \dot{\tilde{\gamma}}^{\lambda}=0
$$


can be expressed as the Levi-Civita geodesics of Riemann gauge $\left(\Gamma \stackrel{\overline{R g}}{{ }_{g}} \Gamma\right)$. It is useful to introduce also a scale covariant parametrization $\gamma(\tau)$ for the (timelike) geodesics such that $g(\dot{\gamma}, \dot{\gamma})=-1$ in all scale gauges (a kind of scale dependent proper time parametrization). This means that one works with scale covariant geodesics for which the weight of the tangent vector field is $w(\dot{\gamma})=-1$.

In local coordinates with $x_{0}=t$ and spacelike indices $i, j, k=1, \ldots 3$ the geodesic equation for a scale covariant timelike geodesic has the same form as in Einstein gravity (cf. e.g. [70, eq. 9.1.2]), but in our case the connection coefficients $\Gamma$ are the Weyl geometric ones $[59$, p. 4]:

$$
\begin{aligned}
\frac{d^{2} x^{j}}{d t^{2}}= & -\Gamma_{00}^{j}+\Gamma_{00}^{0} \frac{d x^{j}}{d t}-2 \Gamma_{0 i}^{j} \frac{d x^{i}}{d t}-\Gamma_{i k}^{j} \frac{d x^{i}}{d t} \frac{d x^{k}}{d t} \\
& +2 \Gamma_{0 i}^{0} \frac{d x^{j}}{d t} \frac{d x^{i}}{d t}+\Gamma_{i k}^{0} \frac{d x^{j}}{d t} \frac{d x^{i}}{d t} \frac{d x^{k}}{d t}
\end{aligned}
$$

In the low velocity, weak field regime the equation of motion reduces to a form well known from Einstein gravity, $\frac{d^{2} x^{j}}{d t^{2}}=-\Gamma_{00}^{j}$. The $\Gamma_{00}^{j}(j=1,2,3)$ are now the coefficients of the Weyl geometric affine connection which differs from the LeviCivita connection of the Riemannian component $g$ by (76). In any scale gauge $(g, \varphi)$ different from Riemann gauge the coordinate acceleration $a$ of freely falling bodies takes up terms from the scale connection, in addition to the Levi-Civita contributions of the Riemannian part $g$ of the metric; in the low velocity, weak field case:

$$
a^{j}=\frac{d^{2} x^{j}}{d \tau^{2}} \approx-\Gamma_{00}^{j}=-{ }_{g} \Gamma_{00}^{j}-{ }_{\varphi} \Gamma_{00}^{j}=a_{g}^{j}+a_{\varphi}^{j}
$$

To the well known metrical acceleration $a_{g}^{j}=-{ }_{g} \Gamma_{o o}^{j}$ known from Riemannian geometry a component $a_{\varphi}^{j}=-{ }_{\varphi} \Gamma_{00}^{j}=g_{00} \varphi^{j}$ induced from the Weylian scale connection is added (cf. Eq. 76). We call these terms the Riemannian acceleration and the additional acceleration due to the scale connection, respectively the scalar field. From the Riemannian (not the Weyl geometric) point of view the latter appears as a "non-metrical" contribution to the acceleration. In Einstein gauge the additional scalar field acceleration for our Lagrangian is (cf. Eqs. 3, 76)

$$
a_{\varphi}^{j}=g_{00} \varphi^{j} \underset{\overline{E g}}{\doteq} g_{00} \partial^{j} \sigma
$$

For the dynamics of freely falling test bodies the exponent $\sigma$ of the scalar field in Riemann gauge functions like an additional gravitational potential. In this respect our approach clearly is a modified gravity model.

\subsection{Einstein gauge and the measured values of observable quantities}

Like in JBD theory one may wonder in IWG gravity which scale gauge expresses the measured values of observable quantities most directly. This may be understood as the 
question for a bridge rule allowing to connect the theoretical (scaling) quantities with the (non-scaling) measured values of empirical quantities. In principle it is possible to formulate such a bridge rule without breaking the scale symmetry just by introducing the scale invariant observable quantity $\hat{X}=\phi^{w(X)} X$ for any scale covariant field quantity $X$ of weight $w(X)$. The scale invariant observable of the scalar field is then $\hat{\phi}=1$. This boils down, up to a constant, to considering the Einstein gauge. This, and the criterion of a best link to Einstein gravity, leads to the

Bridge rule The theoretical values of field quantities of IWG gravity are to be identified with the corresponding empirical values (basically astronomical and astrophysical ones) by going to the Einstein gauge.

In this gauge the coefficient of the Hilbert term (6) is a constant which can be identified with Einstein's gravitational constant,

$$
(\xi \phi)^{2} \underset{\overline{E g}}{\doteq}\left(\xi \phi_{0}\right)^{2}=(8 \pi G)^{-1}\left[\hbar c^{5}\right]
$$

This leads to the closest possible agreement with Einstein gravity in the eEG regime and to Einstein gravity as an exact limit for $\alpha \rightarrow \infty$. Moreover, assuming the biquadratic potential coupling to the Higgs field (8), the expectation value $h_{0}^{2}=\Phi_{0}^{\dagger} \Phi_{0}$ of the Higgs field in the potential minimum (the rest state $\Phi_{0}$ of the Higgs field) also becomes spacetime independent in the Einstein gauge and acquires its experimental value $h_{0}^{2}=$ $v^{2}(v \approx 246 \mathrm{GeV})$. One may like to turn this argument round and argue that the biquadratic coupling to the Higgs field breaks the scale symmetry of the theory. But this leads to a different discussion.

Moreover, we see in Sect. 4.1 that the choice of parameters such that

$$
(\beta \xi)^{-1} \phi_{0} \underset{E g}{\doteq} a_{0}\left[c^{-1} \hbar\right]
$$

with the MOND constant $a_{o} \approx \frac{1}{6} H_{0}[c]$ leads to an agreement with MOND kinematics in the deep MOND regime (but has interesting differences with regard to gravitational lensing). Then the covariant Milgrom equation (29) becomes

$$
{ }_{g} \Delta_{M} \sigma \underset{\overline{E g}}{\doteq} 4 \pi G a_{0}(\rho-3 p)^{(\text {bar })}
$$

Choosing $\beta=6$ fixes the value of $\phi_{0}$ such that

$$
\xi^{-1} \phi \underset{\overline{E g}}{\doteq} \xi^{-1} \phi_{0}=H_{0}
$$

Then $\phi_{0}$ is the geometric mean between the smallest and the largest physically meaningful energy levels we know $\phi_{0}[\hbar]=\sqrt{H_{0}[\hbar] E_{P}}$. For exploring a possible connection to the Higgs portal one may prefer to relax this constraint for $\phi_{0}$ by a different choice of coefficients in (12). 


\section{MG regime and Newton-Milgrom approximation}

\subsection{The modification of Einstein gravity in the MG regime}

The Eqs. $(19,21)$ and $(83)$ show that in Einstein gauge and for conformal coupling of $L_{D \phi^{2}}(\alpha=-6)$, the scale connection part of the Einstein tensor compensates two terms of the energy momentum expression on the r.h.s. of (17): ${ }^{9}$

$$
{ }_{\varphi} G \underset{\overline{E g}}{\doteq} \Theta^{(H)}+\Theta^{\left(D \phi^{2}\right)}
$$

In Einstein gauge and for the MG regime Eq. (17) reduces to an equation for the Riemannian component $g$ of the Weylian metric $(g, \varphi)$ :

$$
{ }_{g} G \underset{\overline{E g}}{\doteq} 8 \pi G T^{(\text {bar })}+\Theta^{(R A Q)}\left[+\Theta^{(V)}\right]
$$

This is a classical Einstein equation for $g$ with the r.h.s. enhanced by an energy momentum term $T^{(\phi)}$ of the scalar field,

$$
\begin{aligned}
& T^{(\phi)} \underset{\overline{E g}}{\stackrel{亠}{\doteq}(8 \pi G)^{-1} \Theta} \\
& \text { with } \Theta \underset{\overline{E g}}{\doteq} \Theta^{\left(D^{2} \phi\right)}+\Theta^{\left(D \phi^{3}\right)}\left[+\Theta^{(V)}\right] .
\end{aligned}
$$

The covariant Milgrom equation $(29)$ and $(35,36)$ teach us that the scalar field has only baryonic matter as its source:

$$
{ }_{g} \Delta_{M} \sigma \underset{\overline{E g}}{\doteq}(4 \pi G) a_{0}(\rho-3 p)_{b a r}
$$

This is an intriguing observation: The scalar field dynamics is sourced by baryonic matter only, while the Riemannian component of the metric has baryonic matter and the scalar field energy-momentum for its source. In this sense one may consider the scalar field as having also the character of dark matter in addition to its being a part of the gravitational structure (Sect. 2.5), although with a peculiar energy-momentum tensor $T^{(\phi)}$ (see Sect. 3.3).

We finally get a twofold modification of Einstein dynamics. The Riemannian acceleration $a_{g}$ is not only due to the baryonic matter, it contains a contribution from the scalar field, which may vanish for special field constellations, see below Eq. (61). Moreover, the total acceleration (33) has an additional component $a_{\varphi}$ due to the scalar field. Both together determine free fall with the total acceleration $a=a_{g}+a_{\varphi}$. The acceleration (33) refers to a weak field, low velocity approximation, but in the general case a similar modification of Einstein gravity holds.

\footnotetext{
9 This cancelling has been overlooked in $[59,60]$. The tensor $\Theta^{(H)}$ contains second order derivative terms comparable to $\Theta^{\left(D^{2} \phi\right)}$ of the present approach (see below, Eq. 58). The dynamics of [59,60] seemed essentially the same as here (corresponding to $\gamma=2$ ) — but at the price of a flawed derivation. See Appendix 6.5
} 


\subsection{Newton-Milgrom approximation}

The Newton approximation of (40) uses a weak field approximation for the Riemannian component of the metric $g$ in Einstein gauge of the form

$$
g \underset{\overline{E g}}{\doteq}+\operatorname{diag}\left(h_{00}, h_{11}, h_{22}, h_{33}\right) \quad \text { with } \eta=\operatorname{diag}(-1,1,1,1)
$$

in which the $h_{j j} \approx 0$ can be neglected, while $h_{00}=-2 \Phi_{N}$ plays the role of the Newton potential [12, p. 153f.]. The approximation to the spatial part of the metric is Euclidean. The first order approximation of $\Gamma$ leads to ${ }_{g} \Gamma_{00}^{j} \approx-\frac{1}{2} \eta^{j j} \partial_{j} h_{00}$. This motivates to set

$$
\Phi_{N}=-\frac{1}{2} h_{00}
$$

then the Riemannian acceleration of (33) acquires the form

$$
a_{g}=-\nabla \Phi_{N}
$$

with the Euclidean gradient operator $\nabla$, like in Newton gravity.

For the Riemannian component of the Ricci tensor we get, at first order in $h[12$, p. 158],

$$
{ }_{g} R_{00} \approx-\nabla^{2} h_{00} .
$$

The Einstein equation (40) solved for the Ricci term is

$$
{ }_{g} R i c=8 \pi G\left(T-\frac{1}{2} \operatorname{tr} T g\right) \text { with } T=T^{(b a r)}+T^{(\phi)},
$$

( $t r$ the trace operator). Because of $-t r T=\rho-\sum_{1}^{3} p_{j}$ its 00-component leads to the Poisson equation for the Newton potential:

$$
\nabla^{2} \Phi_{N} \approx 4 \pi G\left(\rho^{(b a r)}+\sum_{1}^{3} p_{j}^{(b a r)}+\rho^{(\phi)}+\sum_{1}^{3} p_{j}^{(\phi)}\right)
$$

This completes the alignment with Newton dynamics. Here $\rho^{(X)}$ and $p_{j}^{(X)}$ denote the density and pressure components of baryonic matter and the scalar field energymomentum respectively, where $X \in\{b a r, \phi\}$. In the Newton approximation the contribution of the scalar field to the r.h.s. of the Einstein equation is expressed by density and pressure terms analogous to those of classical matter. But here we can no longer expect pressure components $p_{j}=p$ independent of the coordinate direction like for a classical fluid.

The Newton potential and Newton acceleration of the baryonic matter source alone are:

$$
\nabla^{2} \Phi_{N}^{(b a r)}=4 \pi G\left(\rho^{(b a r)}+\sum_{1}^{3} p_{j}^{(b a r)}\right) \quad a_{N}^{(b a r)}=-\nabla \Phi_{N}^{(b a r)}
$$


Analogously for the scalar field contribution:

$$
\nabla^{2} \Phi_{N}^{(\phi)}=4 \pi G\left(2 \rho^{(\phi)}+\operatorname{tr} T^{(\phi)}\right), \quad a_{N}^{(\phi)}=-\nabla \Phi_{N}^{(\phi)}
$$

Here

$$
\rho_{N}^{(\phi \mathrm{st})}=2 \rho^{(\phi)}+\operatorname{tr} T^{(\phi)}=\rho^{(\phi)}+\sum_{1}^{3} p_{j}^{(\phi)}
$$

is the Newton-Poisson source term (st) of the scalar field.

In a weak field regime of a quasi-static scalar field (i.e. one one with relativistically slow velocities/time dependence) considered in the corresponding Newton approximation, the covariant Milgrom equation (29) gets a form like in the classical (i.e. Euclidean/Newtonian) case of the deep MOND dynamics [40]

$$
\nabla \cdot(|\nabla \sigma| \nabla \sigma)=(4 \pi G) a_{0}\left(\rho^{(b a r)}-3 p^{(b a r)}\right) .
$$

Here $\nabla$ denotes the Euclidean gradient operator and "." the Euclidean scalar product. The combination of (47) and (51) will be called a Newton-Milgrom approximation of the relativistic dynamics given by (17)/(40) and (29).

From the empirical evidence acquired in the framework of the classical MOND theory we may conclude that the onset of the MG regime, expressed in quantities of the Newton approximation, occurs in a region in which the norm of the baryonic Newton acceleration comes close to the order of magnitude of the MOND constant $a_{0}[c]$, in short $\left|a^{(b a r)}\right| \leq 10^{2 k} a_{0}$, while the JBD (here Einstein/Newton) regime sets in roughly for $\left|a^{(b a r)}\right| \geq 10^{2(k+l)}$ with, say, $k=l=1 .{ }^{10}$ These conditions serve as provisional distinguishing criteria between the different regimes of the scalar field Lagrangian (see end of Sects. 2.2 and 4.1).

In principle the scalar field contributes two terms $a_{N}^{(\phi)}, a_{\varphi}$ according to Eqs. (49) and (34) to the total acceleration in the MG regime:

$$
a_{t o t}=a_{R}+a_{\varphi}=a_{N}^{(b a r)}+a_{N}^{(\phi)}+a_{\varphi}
$$

But we see in a moment (61) that for simple systems the middle term $a_{N}^{(\phi)}$ vanishes. From (34) we know that the scale connection term is here simply

$$
a_{\varphi}=-\nabla \sigma
$$

In order to compare it with the Newtonian one of the scalar field $a_{N}^{(\phi)}$ we have a closer look at the density and pressure terms of the scalar field in a given weak field regime.

\footnotetext{
$\overline{10}$ We often omit factors $c$ and $\hbar$ in theoretical calculations and plug them in as soon as we approach empirical data. 


\subsection{The scalar field energy-momentum in the Milgrom approximation}

To get an impression of the order of magnitude relations of different entries of $\Theta=$ $\Theta^{\left(D^{2} \phi\right)}+\Theta^{(R A Q)}+\Theta^{(V)}$ in (41) we consider the Newton-Milgrom approximation for a static central symmetric mass distribution of total mass $m, M=G m$, in Euclidean space (radial distance $r$ ). Then the Newton potential of the baryonic source is $\Phi^{(\text {bar })}=$ $-\frac{M}{r}$, while the flat space Milrgrom equation (51) is solved by

$$
\sigma=\sqrt{a_{0} M} \ln \frac{r}{M}
$$

In spherical spatial coordinates (with $x_{1}=r$ ) and the Beltrami-Laplace operator $\nabla^{2}=\partial_{r}^{2}+\frac{2}{r} \partial_{r}$

$$
\partial_{r} \sigma=\frac{\sqrt{a_{0} M}}{r}, \quad \nabla^{2} \sigma=\frac{\sqrt{a_{0} M}}{r^{2}} \quad\left[=-\partial_{r}^{2} \sigma \quad(\text { sic })\right] .
$$

As $\sqrt{a_{0} M} \ll 1$ for $a_{0}<a_{0}$, we find in the MOND region

$$
\left(\partial_{r} \sigma\right)^{2}=\frac{a_{0} M}{r^{2}} \ll \frac{\sqrt{a_{0} M}}{r^{2}}=\nabla^{2} \sigma .
$$

This shows that the entries of $\Theta$ are strongly dominated by the second order derivative terms of $\Theta^{\left(D^{2} \phi\right)}$. The same holds for the entries of the other summands of $\Theta$. We therefore use the approximative energy momentum tensor $(8 \pi G)^{-1} \Theta^{(\text {app })}$ by reducing (23) to its second order derivative terms,

$$
\Theta_{\mu \nu}^{(a p p)} \underset{\overline{E g}}{\doteq} \gamma\left({ }_{g} \nabla_{\mu} \partial_{\nu} \sigma-\frac{1}{2} g_{g} \nabla_{\lambda} \partial^{\lambda} \sigma g_{\mu \nu}\right), \quad T^{(\phi)} \underset{E g}{\approx}(8 \pi G)^{-1} \Theta^{(a p p)},
$$

and work with the approximation $\Theta \underset{E g}{\approx} \Theta^{(a p p)}$ also for the general case of a NewtonMilgrom approximation. In the spatially Euclidean metric this boils down to

$$
\Theta_{\mu \nu}^{(\text {app })} \underset{\overline{E g}}{\doteq} \gamma\left(\partial_{\mu} \partial_{\nu} \sigma-\frac{1}{2} \nabla^{2} \sigma g_{\mu \nu}\right)
$$

This is a peculiar energy-momentum tensor. Its main part has the form of a "vacuum energy" tensor with a coefficient $\left(\nabla^{2} \sigma\right)$ depending, via the Milgrom equation, on the local mass distribution; but superimposed to it we also find an additional pressure term $\partial_{\mu} \partial_{\nu} \sigma$.

For a static or slowly changing scalar field we get the result

$$
\Theta_{00}^{(a p p)} \doteq \underset{\overline{E g}}{\frac{\gamma}{2}} \nabla^{2} \sigma, \quad \rho^{(a p p)} \underset{\overline{E g}}{\doteq} \frac{\gamma}{16 \pi G} \nabla^{2} \sigma
$$


and

$$
\operatorname{tr} \Theta \approx \operatorname{tr} \Theta^{(a p p)} \underset{\overline{E g}}{=}-\gamma \nabla^{2} \sigma .
$$

It follows that the Newton-Poisson source term (50) vanishes,

$$
\rho_{N}^{(\phi \mathrm{st})} \underset{\overline{E g}}{\doteq} 0
$$

From (23) we then read off $2 \Theta_{00}^{\left(D^{2} \phi\right)}-\operatorname{tr} \Theta^{\left(D^{2} \phi\right)} \approx 0$. In the static case and for $g_{00} \approx-1$ this is true already for the unreduced $\Theta^{\left(D^{2} \phi\right)}$, not only for its approximation.

That shows that the r.h.s contribution of the scalar field to the Einstein equation does not essentially enter the Newton approximation for the Riemannian metrical component in Einstein gauge. The only contribution of the scalar field to the acceleration of freely falling bodies is contained in the effects of the scale connection (53), i.e., $a_{\varphi}=-\nabla \sigma$.

The formally calculated mass density which leads to the same acceleration in a Newtonian framework is usually called the "phantom" mass density of modified gravity. Here it is

$$
\rho^{(\text {phant })} \underset{\overline{E g}}{\doteq}(4 \pi G)^{-1} \nabla^{2} \sigma .
$$

For $\gamma=4$ it coincides with the energy density of the scalar field given in (59) and looses its phantom character:

$$
\rho^{(\text {phant })} \underset{\overline{E g}}{\doteq} \rho^{(a p p)} \quad \text { if } \gamma=4
$$

\subsection{Light deflection due to the scalar field}

The scalar field energy-momentum also has crucial repercussions on gravitational light deflection. We check the spatial components of $h_{j j}$ for a weak field approximation with a static Riemannian metrical component $g \underset{\overline{E g}}{\doteq} \eta+h$ like in (42). ${ }^{11}$ For the central symmetric case we express the Minkowski metric $\eta$ and its perturbation $h$ in spatially spherical coordinates $\left(x_{0}, x_{1}, x_{2}, x_{3}\right)=\left(x_{0}, r, \vartheta, \Theta\right)$,

$$
\left.\eta=\operatorname{diag}\left(-1,1, r^{2}, r^{2} \sin ^{2} \vartheta\right), \quad h=\operatorname{diag}\left(h_{00}, h_{11}, 0,0\right)\right)
$$

This is a special case of a spherical symmetric metric $g=\operatorname{diag}\left(-A, B, r^{2} r^{2} \sin ^{2} \vartheta\right)$ with $A=1-h_{00}, B=1+h_{11}$. For $\sigma$ we know the the classical solution (54) of the Milgrom equation, $\sigma=C \ln r$. As usual we consider the approximation of the Einstein equation solved for the Ricci tensor (46).

\footnotetext{
11 For moving matter sources the method of retarded potentials has to be used [57, p. 122f.].
} 
The first two diagonal components of the Ricci tensor (Riemannian component only) are [45, p. 123]

$$
\begin{aligned}
& R_{00}=\frac{A^{\prime \prime}}{2 B}-\frac{A^{\prime} A^{\prime}}{4 A B}-\frac{A^{\prime} B^{\prime}}{4 B^{2}}+\frac{A^{\prime}}{B r} \\
& R_{11}=\frac{A^{\prime} B^{\prime}}{4 A B}-\frac{A^{\prime \prime}}{2 A}+\frac{A^{\prime} A^{\prime}}{4 A^{2}}+\frac{B^{\prime}}{B r}
\end{aligned}
$$

For a weak field approximation we neglect, as usual, the second degree terms in $h$ and its derivatives. From (46), (58) we then get:

$$
\begin{aligned}
& R_{00} \approx-\frac{1}{2}\left(h_{00}^{\prime \prime}+\frac{2}{r} h_{00}^{\prime}\right)=-\frac{1}{2} \nabla^{2} h_{00} \underset{\overline{E g}}{\doteq} 4 \pi G \rho^{(\text {bar })} \\
& R_{11} \approx \frac{h_{00}^{\prime \prime}}{2}+\frac{h_{11}^{\prime}}{r} \underset{\overline{E g}}{\doteq}-4 \pi G \rho^{(\text {bar })}+\gamma \partial_{r}^{2} \sigma
\end{aligned}
$$

The first line is the Newton approximation, $h_{00}=-2 \Phi_{N}$. Adding the two equations leads to

$$
\frac{h_{11}^{\prime}}{r} \underset{\overline{E g}}{\doteq} \frac{h_{00}^{\prime}}{r}+\gamma \partial_{r}^{2} \sigma
$$

For $\sigma=0$ this is the weak field approximation of Einstein gravity with

$$
h_{11}=h_{00}=-2 \Phi_{N} .
$$

But here, for the spherical symmetric solution (54) of the Milgrom equation, (65) turns into

$$
h_{11}^{\prime}=h_{00}^{\prime}-\gamma \frac{\sqrt{a_{0} M}}{r}
$$

and thus $h_{11}=-2 \Phi_{N}-\gamma \sqrt{a_{0} M} \ln r$. For

$$
\gamma=4
$$

this becomes

$$
h_{11}=-2 \Phi_{N}-4 \sigma=-2\left(\Phi_{N}+2 \sigma\right)
$$

Translated in terms of spacelike Cartesian coordinates that means

$$
h=\operatorname{diag}\left(-2 \Phi_{N}, 2\left(\Phi_{N}+2 \sigma\right), 2\left(\Phi_{N}+2 \sigma\right), 2\left(\Phi_{N}+2 \sigma\right)\right)
$$

In a first order approximation with regard to $h$ and its derivatives, the deflection angle $\alpha$ of the spatial wave vector of a small wave package travelling along null geodesics can be expressed in terms of the spatial gradient $\vec{\nabla}$ of $\frac{1}{2}\left(-h_{00}+h_{j j}\right)$ for any $1 \leq j \leq 3$, assuming that all three spatial $h$ are equal [12, p. 288f.]. In the literature often only a pressure-free matter source is discussed. Then $h_{00}=-h_{j j}$, 
and the deflection potential is simply $-h_{00}=2 \Phi_{N}$ In our case with $h_{00} \neq h_{j j}$ the deflection potential is (see Appendix 6.4):

$$
\frac{1}{2}\left(-h_{00}+h_{j j}\right)=2\left(\Phi_{N}+\sigma\right)
$$

With this approximation we arrive at the

Result (a) In the Newton-Milgrom weak field approximation of a spherically symmetric baryonic matter distribution, the invariant potential $\sigma$ of the scalar field contributes to gravitational light deflection via the $h_{j j}, 1 \leq j \leq 3$, in addition to the Newton potential $\Phi_{N}$ which is due only to baryonic matter (both taken twice).

(b) For $\gamma=4$ the effects of the scalar field for light deflection are exactly those of an enhancement of the Newton potential by $\sigma$. This is in quantitative agreement with the additional acceleration induced by the scale connection $a_{\varphi}$ in (53), if one emulates the latter by a formal enhancement of the Newton potential $\Phi_{N}$.

If astronomical observations should indicate a significant difference between the gravitational potentials for trajectories and for light deflection of simple systems in the dark matter sector, our model could accommodate this by lowering or raising $\gamma$; but at the moment there is no reason to do so. ${ }^{12}$

The above result for the central symmetric case suggests the conjecture that also for the general case we can reasonably expect a similar close relation between the potentials for light deflection and for gravitational acceleration, induced by a scalar field with Lagrangian (14) including the term (13).

\section{A relativistic generalization of MOND}

\subsection{Comparison with MOND for simple systems}

A look at (54) and (53) shows that for the central symmetric case the additional acceleration of the scalar field in the Milgrom approximation agrees with the deep MOND acceleration of the classical MOND algorithm. For a comparison between the latter and the present model we introduce the following

Terminology In a Newton approximated relativistic weak field regime with baryonic Newton acceleration $a^{(b a r)}$ we distinguish between the following regions (with, e.g., $k=l=1):^{13}$

(i) the Newton region for $10^{2(k+l)} a_{0} \leq\left|a^{(\text {bar })}\right|$

(ii) the intermediate region $10^{2 k} a_{0} \leq\left|a^{(\text {bar })}\right| \leq 10^{(2(k+l)} a_{0}$

(iii) the MOND region $\quad\left|a^{(\text {bar })}\right| \leq 10^{2 k} a_{0} \quad\left(\leftrightarrow a_{\varphi}=|\nabla \sigma| \leq 10^{k} a_{0}\right)$ containing the deep MOND region $\quad\left|a^{(b a r)}\right| \leq 10^{-2 l} a_{0} \quad\left(\leftrightarrow a_{\varphi} \leq 10^{-l} a_{0}\right)$

\footnotetext{
12 For the relation of virial mass and lensing mass for clusters see Sect. 4.4.

$13 l$ is to be chosen such that $10^{-2 l} a_{0}$ is smaller than the observational errors for accelerations; then the total acceleration of our model in the deep MOND region is well approximated by the additional acceleration of the scalar field $a_{\varphi}$.
} 
The MG regime of our model covers the MOND region (iii); the Newton region (i) falls into the Newton approximated eEG regime, the intermediate regime (ii) is described by the model only formally (Eq. 15).

In the central symmetric case (54) $a_{\varphi} \doteq \sqrt{a_{0} M} r^{-1}$. For a simple, central symmetric system which is not part of a hierarchical gravitational structure (see below) the present model predicts gravitational effects on low velocity free fall trajectories in agreement with MOND dynamics in the deep MOND region. With regard to light bending it differs like Einstein from Newtonian gravity by the factor 2 from non-relativistic MOND calculations and from RAQUAL (see last section).

In the MOND region, i.e. in region (iii), our model can be characterized by the MOND-typical interpolation functions $\mu(x)$ and $v(x)$ [21, eq. (8), (10)]

$$
a_{N}=\mu\left(\frac{a}{a_{o}}\right) a, \quad \text { with } \quad \mu(x) \longrightarrow \begin{cases}1 & \text { for } x \rightarrow \infty \\ x & \text { for } x \rightarrow 0\end{cases}
$$

or the other way round

$$
a=v\left(\frac{a_{N}}{a_{o}}\right) a_{N}, \quad \text { with } \quad v(y) \longrightarrow \begin{cases}1 & \text { for } y \rightarrow \infty \\ y^{-\frac{1}{2}} & \text { for } y \rightarrow 0 .\end{cases}
$$

Here $a_{N}$ stands for the Newton acceleration of the baryonic mass. ${ }^{14}$

For the central symmetric case our acceleration $a=a_{\text {tot }}$ in (52) with vanishing Newton-Poisson term of the scalar field (61) is specified by the interpolation functions

$$
\mu_{w}(x)=1+\frac{1-\sqrt{1+4 x}}{2 x} \text { and } v_{w}(y)=1+y^{-\frac{1}{2}} .
$$

Straight forward calculation (in the approximating Euclidean space) shows that, independent of symmetry conditions, a solution of (51) is given by $\sigma$ with a gradient $\nabla \sigma=-a_{\varphi}$ such that

$$
a_{\varphi}=\left(\frac{a_{0}}{\left|a_{N}^{(b a r)}\right|}\right)^{\frac{1}{2}} a_{N}^{(\text {bar })}=\left(a_{0}\left|a_{N}^{(\text {bar })}\right|\right)^{\frac{1}{2}} \frac{a_{N}^{(\text {bar })}}{\left|a_{N}^{(b a r)}\right|}
$$

The solution of the Milgrom equation (51) is simpler than one might expect. In a first step the linear Poisson equation of the Newton theory is to be solved (48), then an algebraic transformation of type (74) leads to the acceleration given by the nonlinear Poisson equation (51). In this sense our MOND approximation can be solved by means of a quasilinear procedure similar to the so-called "QMOND" approach in the literature [21, eq. (30)].

If our model is realistic the scalar field's energy density and pressure represent properties of a real entity which expresses its mark on the gravitational light deflection. This is a crucial difference to the classical MOND algorithm and to RAQUAL.

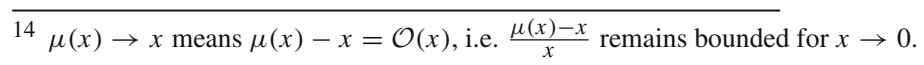


Another important difference results from the following observation: In hierarchical structured gravitating systems, in particular those allowing for Newton-Milgrom approximations at different scales, the scalar field energy $\rho^{(\phi)}$ and the energymomentum tensors $T^{(\phi)}$ of different scales can superimpose. Therefore the scalar field energy density contributions of small scale systems have to be taken into account in the weak field approximation at a higher level. This is different for the pressure terms. In contrast to energy and momentum and the contributions to the scale connection, they are not additive with volume aggregation in a common reference frame and do not allow to form mean values at large scales so easily from their values at small scales.

The other way round, the barycenter of a sufficiently strongly bound subsystem $\mathfrak{A}$ of a larger gravitating system $\mathfrak{B}$ falls freely in the gravitational field of the latter. If for a Newton approximation of $\mathfrak{A}$ the gravitational influences of $\mathfrak{B}$ including its tidal effects can be neglected, the gravitational effects of $\mathfrak{B}$ do not enter the Newton-Milgrom approximation of $\mathfrak{A}$ and can abstracted from, as long as regions with total acceleration (48) above the acceleration of $\mathfrak{B}$ are concerned. This does not hold for gravitationally weakly bound subsystems and the outer regions of strongly bound subsystems. Here already the Newton approximation (48) is precarious and the Milgrom approximation (51) for the scalar field is ill defined. In the present model this seems to be the reason for the external field effect (EFE) of MOND, which has been observed in the dynamics of weakly bound gravitational subsystems in MOND theory [21, sec. 6.3, 6.5.2].

\subsection{A short look at galactic dynamics}

Based on the hypothesis of a covariantly reformulated version of Verlinde's "emergent gravity" (CEG) proposed in [30], Hossenfelder and Mistele study a model for galaxies which leads to MOND-like dynamics with the same interpolation functions as our (73) [31]. They evaluated the data of 2693 measurements referring to 153 galaxies, documented in [39], and find an excellent fit for the radial-acceleration relation of galaxy rotation curves. The empirical check depends only on the interpolation function (73) and therefore applies just as well to the present model (Fig. 1). ${ }^{15}$

In a recent paper [32] the same authors study the hypothesis of a condensate similar to the one of Bherezhiani/Khoury and formulate a model which, in its superfluid phase, leads ("in an idealized limit") to MOND-like dynamics again with the same interpolation function (73). They investigate whether such a model is consistent with empirical data from $[38,44]$ on the rotation curve of the Milky Way. According to their analysis this is the case, if one allows a moderate rescaling of the baryonic mass by a factor $f_{b}=0.8$ which lies in the range of the observational uncertainty. They correctly remark that the result applies also to the approach of covariant emergent gravity (CEG), mentioned above, because it "reduces to the same equations" as the superfluid model (in the mentioned idealized limit). The same is clearly the case for the present scalar field model.

\footnotetext{
15 One has to keep in mind that our $\mu_{w}, v_{w}$ are reliable in the MOND region (iii) only; they do not apply to intermediate region or even the eEG regime.
} 


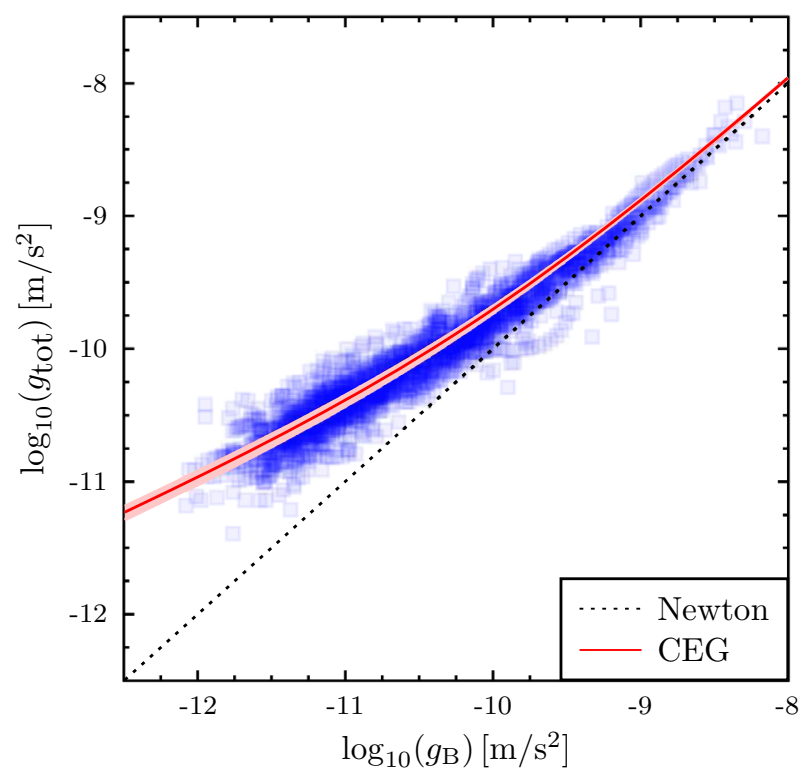

Fig. 1 Blue squares: Observed, total acceleration $g_{\text {tot }}$ compared with acceleration $g_{B}$ due to baryonic mass for 2693 measured data points of 153 galaxies [39]. Red, solid curve $C E G=$ graph of $g_{\text {tot }}$ calculated with $\mu_{w}\left(g_{B}\right)$ (73), according to our notation. Pink shading indicates $1 \sigma$ uncertainty. Dashed, black line: Newtonian gravity without dark matter. Evaluation and figure due to [31] (color figure online)

A crucial difference between the approaches has to be kept in mind. Following Berezhiani/Khoury, the authors assume a pressure less dark matter behaviour of the condensate at the level of galaxy clusters, in fact even already at the outer regions of galaxies (beyond $77 \mathrm{kpc}$ ). As we have seen, this is not the case for our model. Here we have to estimate the aggregation of scalar field energy contributions in the halos of the Newton-Milgom approximation of galaxies in the cluster and to add the effects to the Newton-Milgrom approximation of the cluster as a whole, i.e. we have to study the model's prediction for hierarchical systems.

\subsection{From stars to galaxies and from galaxies to clusters}

Let us shed a first glance at two cases of hierarchical systems in the light of the foregoing remarks: (i) the build-up of galaxies $(\mathfrak{B})$ from stars $(\mathfrak{A})$, (ii) the composition of galaxy clusters $(\mathfrak{B})$ from hot gas $\left(\mathfrak{A}^{\prime}\right)$ and galaxies $(\mathfrak{A})$. In these constellations the NewtonMilgrom approximation for each of the small structures $\mathfrak{A}$ (in its respective barycentric reference system) allows to calculate approximately its scalar field halo with energy momentum $T_{\mathfrak{A}}^{(\phi)}$. After transformation to the barycentric system of the large structure $\mathfrak{B}$, their sum $\sum_{\mathfrak{A}} T_{\mathfrak{A}}^{(\phi)}$ aggregates to a collective scalar field halo which we denote by $\overline{\sum_{\mathfrak{A}} T_{\mathfrak{A}}^{(\phi)}}$. Moreover, the Newton-Milgrom approximation of the global structure in the barycentric reference system of $\mathfrak{B}$ with baryonic mass density $\rho_{\mathfrak{B}}^{(\text {bar })}$ leads to an 
expression $T_{\mathfrak{B}}^{(\phi)}$ for the energy momentum of a global scalar field halo of $\mathfrak{B}$. In the case (i) the aggregation can be understood as an averaging procedure over spacetime regions on an intermediate scale and we may assume that $\overline{\sum_{\mathfrak{A}} T_{\mathfrak{A}}^{(\phi)}} \approx T_{\mathfrak{B}}^{(\phi)}$. In the case (ii) the situation is more complicated because of the intervening hot gas component $\mathfrak{A}^{\prime}$. The relation between the aggregated and the global halos are different in the two cases.

(i) is a well established case in the MOND framework [21, sec. 6.4-6.6]. In principle it can be treated similarly in the present model. Usually a continuity model for the baryonic mass distribution $\rho_{\text {gal }}^{(\text {bar })}$ of a galaxy is used. It is realistic for the gas (neutral hydrogen) inside galaxies, but an idealization for the stellar masses. The MOND acceleration at the galaxy level $a_{M g a l}$ can be calculated from the Newton acceleration $a_{N \text { gal }}$ generated from $\rho_{\text {gal }}^{(\text {bar })}$ by applying the $v$-function of (72) (or the QMOND procedure) [21, p. 58f.]. From our point of view, the superposition of the scalar field halos (58) of the single stars $\sum_{*} T_{*}^{(\phi)}$ reappears in this procedure aggregated in the form of a continuity representation of the scalar field halo of the galaxy as a whole $T_{g a l}^{(\phi)}$. It is (calculated in the Newton-Milgrom approximation in the galacto-barycentric system). This procedure presupposes a silent substitution of the spatial average density $\overline{\sum_{*} T_{*}^{(\phi)}}$ of the scalar field halos of the stars $\sum_{*} T_{*}^{(\phi)}$ by the global model for the galactic halo $T_{\text {gal }}^{(\phi)}$. The acceleration effects of the scalar field are given by the scale connection at the galaxy level $\varphi_{g a l}$ and the corresponding acceleration $a_{\varphi \text { gal }}$. In the usual MOND approach the latter can be expressed by a phantom mass density formally associated to the MOND-acceleration field $a_{M g a l}$. In our approach this is different. The aggregated scalar field halos of the stars and the overall scalar field halo of the galaxy seem to be more or less equal, $\overline{\sum_{*} T_{*}^{(\phi)}} \approx T_{\text {gal }}^{(\phi)}$, with $T_{\text {gal } 00}^{(\phi)}=\rho_{\text {gal }}^{(\phi)}$ the energy density of the galaxy's scalar field halo in the galacto-barycentric reference system. In our model $\rho_{\text {gal }}^{(\phi)}$ expresses a veritable energy density (63); it is no phantom.

Two questions have to be posed here: ${ }^{16}$

(i-a) Is the aggregation procedure $\overline{\sum_{*} T_{*}^{(\phi)}} \approx T_{\text {gal }}^{(\phi)}$ consistent?

(i-b) Is the result compatible with empirical evidence? This is more or less the same as asking whether our interpolation functions (73) are empirically acceptable at the level of galaxies.

Reasons to be optimistic with regard to the second question are given in a comparable constellation in [60, sec. 5.3], but a detailed answer can only be given by astronomers. The first question is of a more theoretical nature, but a positive answer to the second one might be taken as an empirical indicator for a positive answer to the first one.

Case (ii) is more subtle. Even if we leave aside the difficulties in gaining realistic mass density profiles for the hot gas in clusters $\rho_{\text {clust }}^{\text {(gas) }}$ and a continuity model for the star mass density profile $\rho_{\text {clust }}^{(*)}$, both adding up to the baryonic mass density $\rho_{\text {clust }}^{\text {(bar })}=\rho_{\text {clust }}^{(\text {gas })}+\rho_{\text {clust }}^{(*)}$ of the cluster ${ }^{17}$, there arise new problems at the cluster

\footnotetext{
16 Of course there are more questions to ask; for example: Can relativistic corrections to the external field effect for the solar system in the Milky Way galaxy be estimated and tested (cf. [21, p. 57])?

17 See, e.g., $[53,77,78]$.
} 
level. In the usual calculation of the MOND acceleration $a_{M c l u s t}$ from the baryonic density $\rho_{\text {clust }}^{\text {(bar) }}$ via its Newton acceleration $a_{N \text { clust }}$ like in (i), the dominance of the hot gas mass over star/galaxy mass leads to a lower contribution of the star mass to the MOND acceleration $a_{\text {Mclust }}$ and the associated phantom mass $\rho_{\text {Mclust }}^{\text {(phant) }}$ at the cluster level than one would expect if the galaxies were considered on their own (without the hot gas). The same holds for the additional acceleration $a_{\varphi, c l u s t}$ at the cluster level in our approach — and here it has consequences.

Each of the galaxies is a subsystem $\mathfrak{A}$ freely falling in the gravitational field of the cluster $\mathfrak{B}$ and has a scalar field halo which can be calculated in the Newton-Milgrom approximation of the corresponding galactocentric reference system. The totality of them superposes to $\sum_{g a l} T_{g a l}^{(\phi)}$. If we want to estimate its averaged value $\overline{\sum_{g a l} T_{\text {gal }}^{(\phi)}}$ over intermediate distance scales (between galactic scale and cluster scale) in the continuity model, we have to consider the idealized global mass distribution $\rho_{\text {clust }}^{(*)}$ of the galaxies and calculate the corresponding Newton-Milgrom approximation at the cluster level separately from the hot gas. This leads to a scalar field halo of the stars at the cluster level $T_{c l u s t}^{(\phi *)}$ and a scale connection $\varphi_{*}$ (in the cluster barycentric reference system and in Einstein gauge) with the corresponding acceleration denoted by $a_{\varphi *}$. If we are optimistic, we may assume that the continuity calculation gives an estimate for the averaged scalar halos of all the galaxies like in the case (i), $\overline{\sum_{\text {gal }} T_{\text {gal }}^{(\phi)}} \approx T_{\text {clust }}^{(\phi *)}$. Similarly the scalar field halo of the hot cluster gas $T_{\text {clust }}^{(\phi \text { gas })}$ can be estimated by the Newton-Milgrom approximation with the baryonic content $\rho_{\text {clust }}^{(\text {gas })}$ alone. It contributes a term $\varphi_{\text {gas }}$ to the scale connection and induces the additional acceleration $a_{\varphi \text { gas }}$ according to (34). The accelerations $a_{\varphi *}$ and $a_{\varphi \text { gas }}$ are due to the scale connection. They are not phantom and their effects add up. They are closely related to the corresponding (approximate) scalar field energy densities $\rho_{\text {clust }}^{(\phi *)}$ and $\rho_{\text {clust }}^{(\phi \text { gas })}$ calculated according to eqs. (59), (62), (63). Of course, the Newton approximation at the cluster level leads to a Newtonian acceleration $a_{N}$ clust sourced by the total cluster mass density $\rho_{\text {clust }}^{(\text {bar })}$. The total acceleration in the cluster model is then

$$
a_{\text {clust tot }}=a_{N \text { clust }}+a_{\varphi g a s}+a_{\varphi *}
$$

If one wanted to emulate the same acceleration in a Newtonian model, the Eqs. (62) and (63) show that one has to take account of the combined mass density of baryonic origin and the two constitutive parts of the scalar field halo;:

$$
\rho_{\text {clust }}^{\text {tot }}=\rho_{\text {clust }}^{(\text {bar })}+\rho_{\text {clust }}^{(\phi \text { gas })}+\rho_{\text {clust }}^{(\phi *)}
$$

The lensing of clusters arises partially from the weak field approximation of the global cluster model with the hot gas as baryonic source which incorporate effects from the cluster scalar field halo in addition to the Newton potential. But the total lensing mass of the cluster incorporates also the contributions due to the micro-lensing effects of all the halos of the galaxies. Here the Newton-Milgrom approximation at the cluster level has to be complemented by an estimate of the aggregated effects of the galactic scalar field halos, which are not taken into account for the global continuity model 


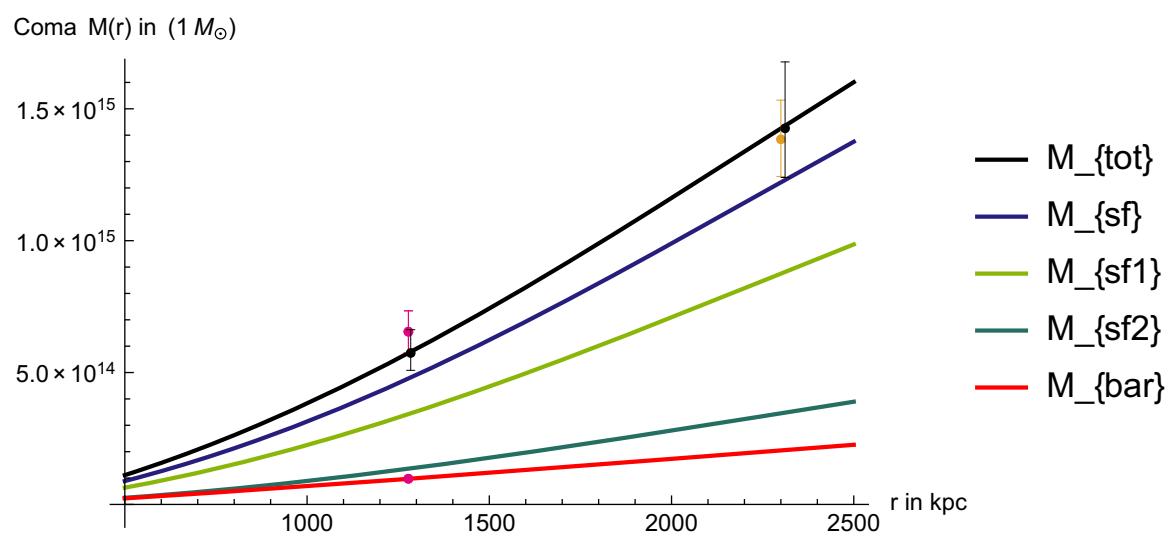

Fig. 2 Development of mass contributions in the model for the Coma cluster, depending on the distance $r$ from the center: Baryonic mass (hot gas and galaxies) $M_{b a r}$ (red) interpolated by a $\beta$-model (see [59]), contribution of the scalar field halo $M_{s f 2}$ of the galaxies (dark green), the hot gas $M_{s f 1}$ (bright green), the combined scalar field halo $M_{s f}=M_{s f 1}+M_{s f 2}$ (blue), and the total mass $M_{t o t}=M_{s f}+M_{b a r}$ (black). Observational values for the baryonic mass (red dot) and the virial mass at $r_{500}=1280 \mathrm{kpc}$ (red error interval), the latter also at $r_{200}=2300 \mathrm{kpc}$ (orange error interval). Black error intervals at $r_{500}, r_{200}$ indicate model spread resulting from the variation of observational input data (color figure online)

with $\rho_{\text {clust }}^{\text {bar }}$ as baryonic source because of the dominance of the hot gas component in the latter. In these respects the present model differs considerably from the original MOND estimates at cluster level (Fig. 2).

\subsection{A heuristic discussion of cluster dynamics}

If the above optimistic approach for estimating the averaged scalar field halo of the galaxies is justified, our model may be able to explain cluster dynamics without additional dark matter. A heuristic check with observational data of $17+2$ clusters taken [77,78], evaluated like in [59] for a similar, although in its justification problematic, model gave encouraging results. For 15 of the 17 outlier reduced clusters the observational errors and the model spread overlap (for the remaining two a minor extension leads to overlapping). Among the 15 is the famous Coma cluster with an observational value for the total mass $M_{500}=6.55^{ \pm 2.36} 10^{14} M_{\odot}$ inside the reference distance $r_{500}=1278 \mathrm{kpc} .{ }^{18}$ The total mass equivalent of $\rho_{\text {clust }}^{\text {tot }}$, calculated in our model including the scalar field halos of the hot gas and the galaxies and integrated up to the reference distance, is $M_{t o t}(500) \approx 5.7_{-0.7}^{+0.9} \cdot 10^{14} M_{\odot}$. That compareswell with the observational value and leaves no mass gap like in the usual MOND approach,

\footnotetext{
$18 r_{500}$ is the distance from the cluster center, at which the total mass density, reconstructed in the framework of a dark matter paradigm from observational data, has fallen down to $500 \rho_{\text {crit }}$, with $\rho_{\text {crit }}$ the critical density of cosmology. $M_{500}$ is the values for the total mass inside the respective reference distance, determined from observational data in the framework of the dark matter paradigm.
} 
see figure 1 . This may be a motivation for more detailed, not only heuristic investigations. $^{19}$

For a better understanding of cluster dynamics questions similar to (i-a, i-b) have to be tackled:

(ii-a) Is the above mentioned ( "optimistic") estimate of the collective scalar field halos of the galaxies in the continuity model $\overline{\sum_{\text {gal }} T_{\text {gal }}^{(\phi)}} \approx T_{\text {clust }}^{(\phi *)}$ consistent with, e.g., numerical simulations?

(ii-b) Are the effects of the superposition of scalar field halos of all the galaxies in the cluster and the halo of the hot gas (of course considered in addition to the Newton potential) generated by the total baryonic mass sufficient to explain the observational data of clusters?

Aside from these questions, let us shed a short glance at the bullet cluster 1E065756. It is often interpreted as providing direct evidence in favour of particle dark matter and of ruling out alternative gravity approaches. This argument does not apply to the present model. The energy content of the scalar field halos of the colliding clusters endows them with inertia of their own. The shock of the colliding gas exerts dynamical forces on the gas masses only, not directly on the scalar field halos. We can expect that, during the encounter, the halos will roughly follow the inertial trajectories of their respective clusters before collision, and they will continue to do so for a while. Only after a certain time delay a re-adaptation of the mass systems and the respective scalar field halos can take place. Clearly the MOND-approximation of the present model is unable to cover such violent dynamical processes. It describes only the relatively stable states before collision and-in some distant future - after collision. But a separation of halos and gas masses for a (cosmically "short") period is to be expected, just like in the case of a particle halo with appropriate clustering properties .

For the time being, the cluster 1E0657-56 does not help to decide between the overarching alternative research strategies, particle dark matter or alternative gravity; all the more so for modification by a scalar field carrying a non-negligible amount of energy-momentum like in the present approach. It may be able to do so, once the dynamics of gas and of the halos has been modelled with sufficient precision in both approaches. Only then a proper comparison can be made; but that is an overtly complicated task. It seems more likely that other types of observational evidence will offer a simpler path to a differential evaluation of the two strategies and help clarifying the alternative.

Here, even more than in the case of the galaxy dynamics, a reliable judgement on the empirical feasibility of the model can only be given by astronomers. For the moment we have to content ourselves with the theoretically intriguing properties of the energy momentum tensor of the scalar field, which may indicate a new route towards solving the missing mass problem for clusters.

19 By several reasons the calculations of $[59,61]$ are problematic; see Appendix 6.5. But the numerical results do not differ strongly from the present ones based on a reliable derivation. For example the value of the total mass equivalent of the Coma cluster given in [61, table 4$]$ is $M_{t o t}(500) \approx 5.7_{-0.7}^{+1} \cdot 10^{14} M_{\odot}$. 


\section{Discussion and outlook}

The framework of the model studied in this paper can be viewed as a kind of generalized Jordan-Brans-Dicke approach, but it becomes more transparent if it is formulated in terms of integrable Weyl geometry. It leads to a modification of Einstein gravity with a scale invariant Einstein equation (17). We have assumed that the scalar field appears in two phases governed by different Lagrangians in the eEG regime and the MG regime. In the first one the scalar field is governed by a usual quadratic kinetic Lagrange term with $\alpha \gg 1$; here the dynamics agrees effectively with Einstein gravity. The present paper concentrates on the MG regime in which Einstein gravity is modified for very very weak gravitational field constellations. Here the scalar field Lagrangian consists of a cubic kinetic term typical for MOND-like behaviour (11), a conformally coupled quadratic kinetic term, and a second order kinetic self-interaction term important for the self-energy of the scalar field (13).

In the Einstein gauge of the Weylian metric ("Einstein frame") the gravitational equation of the MG regime can be brought into the form of an Einstein equation (40) for the Riemannian component $g$ of the Weylian metric. The scalar field $\phi$, in Riemann gauge ("Jordan frame") ${ }^{20}$ written as $\tilde{\phi}=\phi_{0} e^{-\sigma}$, is governed by a covariant version of Milgrom's nonlinear Poisson equation for the exponent $\sigma$ (29). Accordingly, for a weak field, quasi-static constellation one has to consider weak field approximations for the two constituents $g$ and $\varphi$ of the Einstein gauged Weylian metric, where $\varphi=d \sigma$. The Einstein equation for $g$ leads to a Newton approximation (47) which is sourced by the baryonic matter and the scalar field. Surprisingly, the additional source term of the scalar field (50) vanishes for our Lagrangian (61). The scalar field equation for $\sigma$ simplifies to the classical Milgrom equation in Euclidean space (51). Both together constitute the Newton-Milgrom weak field approximation of the present theory.

In this approximation the kinematics of freely falling test bodies (32) is influenced by the invariant potential $\sigma$ through a modification of the affine connection in Einstein gauge (34). For an appropriate choice of the coefficients (36) this leads to a MONDlike phenomenology, with interpolating functions (73) taken into closer considerations already by Hossenfelder/Mistele based on a different Lagrangian approach. Of course, the latter are valid only in the MG regime. In the eEG regime, the influence of the scalar field is negligible; as already said it is effectively governed by Einstein gravity. In the MG regime the role of the scalar field as a source term for the Einstein equation (40) and for the weak field approximation used for calculating light deflection (64) has important consequences for gravitational lensing, which is different from both classical MOND and RAQUAL. For the central symmetric case it is given by (69).

For hierarchical systems like galaxies, formed from stars and interstellar gas, the scalar field of the global system may be approximated by the averaged scalar field of the stars as constituent parts. For clusters, formed from galaxies and hot gas, this is different because of the dominance of the hot gas in the total baryonic matter. Here the scalar field halos of the galaxies have to be taken into account in addition to the halo of the hot gas. This is clear for the lensing mass of the cluster and can be reasonably

20 See fn. 1. 
assumed also for its virial mass. This may suffice to close the mass gap arising in the usual MOND approach to cluster dynamics.

A series of open questions remains:

On the theoretical level the contribution of the scalar field to the light deflection potential has to be worked out for the general case. Moreover it remains to be checked, whether the propagation velocity of scalar field excitations is different from RAQUAL because of the second order kinetic term (13). And even if not, the difficult question of group velocity versus phase velocity had to be decided, before one comes to a final judgement of the physical feasibility of the approach.

On the empirical side there are important questions of adequacy at the scale of galaxies and of galaxy clusters (cosmological questions belong to an epistemologically different class, see below): (i-b) Can the interpolating functions given in (73), which arise from the additive overlay of the scalar field effects to the baryonic Newton dynamics, reproduce the successes of the classical MOND algorithm for galaxy rotation curves? Encouraging first positive results are given in [31,32]. (ii-b) Are the estimates of the total virial mass and the lensing mass of clusters in agreement with observational data, possibly even without assuming additional dark matter?

Another class of questions refers to a possible material underpinning for the Lagrangian (presupposing a positive answer to the questions of empirical adequacy). It seems unlikely that the scalar field $\phi$ of the model represents a fundamental field. If it is physical, it is much more likely that it expresses some collective state comparable to the superfluid approach to DM/MG. But the energy-momentum tensor (58) shows more similarities with what is usually considered as a "dark energy" tensor than with dark matter. The different subcategories of the dark sector seem to be moved closer together than is usually thought. This may indicate a problem for the possibility of bringing the present scalar field model in closer relation with the superfluid approach; but the question remains open.

From a different perspective, the biquadratic potential of $\phi$ with the Higgs field (8) can be used to establish a (weak) connection between our field and the Higgs portal. In this case the hierarchy factor $\eta$ in the potential has to be explained and the question of the fundamental constituents for the collective state function $\phi$ is posed. A-very speculative_-possibility is studied in approaches like [13,27,43], for which the integrable scale connection, and its scalar field $\phi$, arises as a collective limiting case of a non-integrable scale connection $\varphi$ with a "true" Weyl vector boson is being studied. This may also lead to different view of the distinction between JBD and MG regime assumed here: If the scalar field arises as a collective excitation state of a quantum field and vanishes in higher curvature regions of space-time, the scalar field in the eEG regime may be just a formal fiction, and the non-MG regime could turn out to be governed by Einstein gravity per se. In this case the Lagrangian density of the scalar field in (14) for the eEG regime would have to be substituted by a Lagrange constraint $\sum_{\nu} \lambda_{\nu} D_{\nu} \phi$ for the Einstein regime, setting $D_{\nu} \phi=0$ (for all $v$ ) and enforcing the identity of Riemann gauge with the Einstein gauge [27].

Questions of cosmology have not been dealt with here. It seems not yet clear, whether the MG regime of the present model extends to the cosmological scale, and if so which consequences would arise for cosmological models. For studying such questions it should be taken into account that the cosmological problems leading to DM and of 
MG have a different epistemic source than the astrophysical ones dealt with here. Cosmological models have an inherent epistemic ambiguity. They try to scientifically represent the material world as a whole and thus are always in the danger of overstretching the scientific method. Although the present standard model of cosmology can proudly claim many impressive successes, any further going contention of the predominance for cosmological tests over those from astrophysical observations in more "nearby" regions (say redshift $z<3$ ) indicates lacking critical self-reflection of the epistemic status of the cosmological model. It should be rejected or passed over in silence.

The recent Hubble telescope data on the Hubble parameter, which indicate a $4 \sigma$ discrepancy between (cosmologically) model-independent, direct measurements of $H_{0}$ from the nearby universe and predictions from the "early" universe made on the background of the $\Lambda C D M$-model of cosmology [54], may be a warning sign. The authors of the study draw the conclusion "A new feature in the dark sector of the Universe appears increasingly necessary to explain the present difference in views of expansion from the beginning to the present" [54, preprint p. 18]. To give this quote here does not mean claiming the status of such a "new feature of the dark sector" for scalar field halos of the present approach. It rather serves the purpose of underpinning the above mentioned choice of priority for "nearby" astrophysical observational evidence over cosmological criteria which presuppose the $\Lambda C D M$-model.

In any case, our model also has some interesting features on a general, so to speak philosophical level. It demonstrates that the possibilities for the elaboration of models for the explanation of astrophysical DM phenomena starting from a MG approach in the framework of a classical geometric setting and a simple field content are not yet exhausted. Just to the contrary, our relatively simple scalar field model is based on a natural, moderate generalization of Riemannian geometry; it does not need to impute artificially looking structures for the physical geometry of space-time. Its kinetic selfinteraction term tends to undermine the presently dominant dichotomy between spacetime and the dark sector, a bit like the superfluid approach does with regard to the DM-gravity dichotomy [37]. In contrast to the latter the energy momentum tensor of the scalar field resembles dark energy more than (dark) matter. We should keep these strange properties of the energy-momentum tensor in mind. They may be a sign that the present approach has a value as a formal model only; if not, they would seem to indicate that a greater shift in the ontology of the dark sector becomes necessary.

Acknowledgements Open Access funding provided by Projekt DEAL. Many of the general reflections in this paper, and the motivation for writing it, are due to the common work in the project-group A3 "LHC and gravity" of the interdisciplinary group Epistemology of the Large Hadron Collider generously funded by the German $D F G$ and the Austrian $F W F$. T. Scholz made me aware of the recent important work of Riess et al.; D. Lehmkuhl of the Geroch-Jang theorem. An anonymous referee encouraged me to discuss the bullet cluster and the question of the intermediate region between the effective Einstein and the modified gravity regime.

Open Access This article is licensed under a Creative Commons Attribution 4.0 International License, which permits use, sharing, adaptation, distribution and reproduction in any medium or format, as long as you give appropriate credit to the original author(s) and the source, provide a link to the Creative Commons licence, and indicate if changes were made. The images or other third party material in this article are included in the article's Creative Commons licence, unless indicated otherwise in a credit line to the material. If 
material is not included in the article's Creative Commons licence and your intended use is not permitted by statutory regulation or exceeds the permitted use, you will need to obtain permission directly from the copyright holder. To view a copy of this licence, visit http://creativecommons.org/licenses/by/4.0/.

\section{Appendix}

\subsection{Weylian metric, derivative operators, curvatures, Einstein tensor}

There are many introductions to Weyl geometry, among them the classics [8,20,48,7173]. ${ }^{21}$ Here follows a short introduction with particular emphasis on the conventions and notations used in this paper.

A Weyl geometric structure can be specified in a scale-gauge dependent manner by a pair $(g, \varphi)$ of a semi-Riemannian metric $g=g_{\mu \nu} d x^{\mu} d x^{\nu}$, representing the Riemannian component of the Weylian metric and a differential 1-form $\varphi=\varphi_{\nu} d x^{\nu}$ representing the scale connection in the chosen scale-gauge. A change of scale (or gauge) is given by conformal rescaling $\tilde{g}=\Omega^{2} g$ accompanied by the gauge transformation $\tilde{\varphi}=\varphi-d \log \Omega$. The scale invariant affine connection $\Gamma$ (in coefficients $\Gamma_{\mu \nu}^{\lambda}$ ) of Weyl geometry is a sum $\Gamma=\Gamma(g)+\Gamma(\varphi)$ of the Levi-Civita connection $\Gamma(g)$ of $g$ and a part depending on the scale connection, which in coefficients is given by

$$
\Gamma(\varphi)_{\mu \nu}^{\lambda}=\varphi_{\mu} \delta_{\nu}^{\lambda}+\varphi_{\nu} \delta_{\mu}^{\lambda}-g_{\mu \nu} \varphi^{\lambda}
$$

To avoid clumsy expression we also use a notation with pre-sub-scripts $g$ and $\varphi$ like ${ }_{g} \Gamma=\Gamma(g)$ and ${ }_{\varphi} \Gamma=\Gamma(\varphi)$ etc. $\Gamma$ defines a covariant derivative operator $\nabla=\nabla(\Gamma)$ different from the covariant derivative with regard to the Levi-Civita of the Riemannian component, also denoted by pre-sub-script ${ }_{g} \nabla=\nabla(\Gamma(g))$.

For scale covariant fields $X$ of weight $w(X)=w$ (i.e. $X \mapsto \tilde{X}=\Omega^{w} X$ for a change of scale by $\Omega$ like above) the scale covariant derivative $D$ is given by $D X=$ $\nabla X+w \varphi \otimes X$, e.g. for a vector field $D_{\mu} X^{v}=\nabla_{\mu} X^{v}+w \varphi_{\mu} X^{v}$, for a scalar field $D_{\mu} X=\partial_{\mu} X+w \varphi_{\mu} X$ etc. It is important to distinguish the three derivations

$$
{ }_{g} \nabla X, \quad \nabla X, \quad D X
$$

for scale covariant fields (scalar, vector or tensor). Lifting and lowering of indices, i.e. transformations between tangent vector components and its duals, are given by the Riemannian component $g$ in a scale gauge and thus change the scale weight of a field, e.g. $w\left(X^{\mu}\right)=w\left(g^{\mu \nu} X_{v}\right)=w\left(X_{v}\right)-2$.

The Weyl geometric Riemann tensor is defined as Riem $=\operatorname{Riem}(\Gamma)$ and thus scale invariant. The same holds for the Ricci tensor $\operatorname{Ric}=\operatorname{Ric}(\Gamma)$, while the scalar curvature (Ricci scalar) $R$ uses lifting of indices and is thus of weight $w(R)=-2$. Like

\footnotetext{
${ }^{21}$ Mor recent ones can be found in [9,33], [68, chap. IX], [19, appendix A] and [49] (difficult to access). For selected aspects see [15] and [43, sec. 4]. Integrable Weyl geometry is presented in [1,16,52,55], [58, sec. 2.1]. Be aware of different conventions for the scale connection. Expressions for Weyl geometric derivatives and curvature quantities are derived in $[28,76]$ and $[41$, App.]. For a more mathematical perspective consult $[10,22,25,29]$.
} 
for the affine connection one often needs to compose the Weyl geometric curvature quantities from their Riemannian counter parts (depending on $g$ in one gauge only) and a scale connection part; we write

$$
\text { Riem }={ }_{g} \text { Riem }+{ }_{\varphi} \text { Riem etc. }
$$

The Einstein tensor $G=$ Riem $-\frac{1}{2} R g$ is similarly (de-)composed

$$
G={ }_{g} G+{ }_{\varphi} G
$$

The curvature of the scale connection $\varphi$ is given by the exterior differential $d \varphi$. If it vanishes $\left(\partial_{\mu} \varphi_{\nu}-\partial_{\nu} \varphi_{\mu}=0\right)$, the Weylian metric is locally integrable, i.e., at least for simply connnected regions it can be brought into the form of a Riemannian metric by chosing a gauge with $\tilde{\varphi}=0$. This is called the Riemann gauge.

\subsection{Some useful formulas}

For the Ricci tensor of Weyl geometry Ric $={ }_{g} R i c+{ }_{\varphi}$ Ric, the scalar curvature $R={ }_{g} R+{ }_{\varphi} R$ and the Einstein tensor $G={ }_{g} G+{ }_{\varphi} G$ the following relations hold in dimension $n$ for any scale gauge

$$
\begin{aligned}
{ }_{\varphi} R_{\mu \nu} & =(n-2)\left(\varphi_{\mu} \varphi_{\nu}-{ }_{g} \nabla_{(\mu} \varphi_{\nu)}\right)-\left((n-2) \varphi_{\lambda} \varphi^{\lambda}+{ }_{g} \nabla_{(\lambda} \varphi^{\lambda)}\right) g_{\mu \nu} \\
{ }_{\varphi} R & =(n-1)(n-2) \varphi_{\lambda} \varphi^{\lambda}-2(n-1){ }_{g} \nabla_{\lambda} \varphi^{\lambda} \\
{ }_{\varphi} G_{\mu \nu} & =(n-2)\left(\varphi_{\mu} \varphi_{\nu}-{ }_{g} \nabla_{(\mu} \varphi_{\nu)}+\left(\frac{n-3}{2} \varphi_{\lambda} \varphi^{\lambda}+{ }_{g} \nabla_{(\lambda} \varphi^{\lambda)}\right) g_{\mu \nu}\right)
\end{aligned}
$$

In our case

$$
{ }_{\varphi} G_{\mu \nu} \underset{\overline{E g}}{\doteq} 2\left(\partial_{\mu} \sigma \partial_{\nu} \sigma-{ }_{g} \nabla_{(\mu} \partial_{\nu)} \sigma\right)+\left(\partial_{\lambda} \sigma \partial^{\lambda} \sigma+2 \nabla_{(\lambda} \partial^{\lambda} \sigma\right) g_{\mu \nu}
$$

For $\tilde{\phi} \underset{R g}{\doteq} \phi_{0} e^{-\sigma}$ the following holds:

$$
\begin{aligned}
D_{\mu} \phi & =\partial_{\mu} \phi-\varphi_{\mu} \phi \underset{\overline{E g}}{\doteq}-\varphi_{\mu} \phi_{0} \underset{\overline{E g}}{\doteq}-\phi_{0} \partial_{\mu} \sigma \\
D_{\lambda}\left(|D \phi| D^{\lambda} \phi\right) & \underset{\overline{E g}}{\doteq}-\phi_{0} D_{\lambda}\left(|D \phi| \partial^{\lambda} \sigma\right)
\end{aligned}
$$

And because of ${ }_{\varphi} \Gamma_{\mu \nu}^{\mu}=4 \varphi_{\nu}$

$$
\begin{aligned}
D_{\mu} D^{\mu} \phi & =\nabla_{\mu} D^{\mu} \phi-3 \varphi_{\mu} D^{\mu} \phi={ }_{g} \nabla_{\mu} D^{\mu} \phi+\varphi_{\mu} D^{\mu} \phi \\
& \doteq-\tilde{\bar{\phi} g}\left({ }_{g} \nabla_{\mu} \partial^{\mu} \sigma-\partial_{\mu} \sigma \partial^{\mu} \sigma\right) \\
& \doteq \phi_{0}\left({ }_{g} \nabla_{\mu} \partial^{\mu} \sigma+\partial_{\mu} \sigma \partial^{\mu} \sigma\right)
\end{aligned}
$$


As $|\nabla \sigma|=\epsilon_{\sigma} \partial_{\sigma} \partial^{\sigma}$ and therefore $w(|\nabla \sigma|)=-2$, we get

$$
\begin{aligned}
D_{\lambda}\left(|\nabla \sigma| \partial^{\lambda} \sigma\right) & ={ }_{g} \nabla_{\lambda}\left(|\nabla \sigma| \partial^{\lambda} \sigma\right)+{ }_{g} \Gamma_{\lambda \nu}^{\lambda}|\nabla \sigma| \partial^{\nu} \sigma-3 \varphi_{\lambda}|\nabla \sigma| \partial^{\lambda} \sigma \\
& ={ }_{g} \nabla_{\lambda}\left(|\nabla \sigma| \partial^{\lambda} \sigma\right)+\varphi_{\lambda}|\nabla \sigma| \partial^{\lambda} \sigma
\end{aligned}
$$

For the variation $\frac{\delta L_{D \phi^{3}}}{\delta \phi}$ the following modules are helpful:

$$
\begin{aligned}
\frac{\partial L_{D \phi^{3}}}{\partial \phi} & =-2 \phi^{-1} L_{D \phi^{3}}, \quad \frac{\partial L_{D \phi^{3}}}{\partial\left(D_{\lambda} \phi\right)}=-2 \beta \xi^{3} \phi^{-2}|D \phi| D^{\lambda} \phi \\
D_{\lambda} \frac{\partial L_{D \phi^{3}}}{\partial\left(D_{\lambda} \phi\right)} & =-2 \beta \xi^{3} \phi^{-2} D_{\lambda}\left(|D \phi| D^{\lambda} \phi\right)+6 \phi^{-1} L_{D \phi^{3}}
\end{aligned}
$$

\subsection{Geroch-Jang theorem}

In Einstein gravity (EG) the principle of geodesic motion of test particles is supported by the following theorem of Geroch and Jang [26].

\section{Theorem Geroch-Jang}

After appropriate re-parametrization, a smoothly embedded timelike curve $\gamma$ in an oriented Lorentzian manifold $(M, g)$ is a geodesic if the following holds: For any open neighbourhood $U$ of $\gamma$ (more precisely its image/trace) there is a smooth symmetric 2-form $T_{a b}$ with support in $U$, which does not vanish identically, is covariantly conserved, and satisfies the strong dominant energy condition in the following sense: For every timelike covector field $\xi_{a}$ the vector field $T^{a b} \xi_{a}$ is timelike at any point and $T^{a b} \xi_{a} \xi_{b} \geq 0$.

This theorem may reasonably be interpreted as implying the geodesic principle: Sufficently small test bodies move along timelike geodesics. It is easily imported into the integrable Weyl geometric (IWG) framework by the following argument [36].

Theorem Geroch-Jang (IWG)

Let $\gamma: I \longrightarrow M$ be a smoothly embedded timelike curve in an oriented integrable Weylian manifold $(M,[(g, \varphi)])$ satisfying the following properties: For any open neighbourhood $U$ of $\gamma$ (more precisely its image/trace) there is a smooth, not identically vanishing, symmetric scale covariant 2 -form $T_{a b}$ of weight $w\left(T_{a b}\right)=-2$ with support in $U$, which satisfies the conditions (derivation operators $\nabla$ and $D$ as in (77):

(i) $T$ is scale covariantly conserved, $D_{a} T^{a b}=0$.

(ii) $T$ satisfies the strong dominant energy condition in any gauge. ${ }^{22}$

Then the curve can be re-parametrized, $\tilde{\gamma}: I \longrightarrow M$, as a Weyl geometric geodesic. ${ }^{23}$

The proof is easy. Obviously the conditions (i) and (ii) hold in any scale gauge, if they are satisfied in one. Go to Riemann gauge. Then the conditions of the Riemannian

\footnotetext{
${ }^{22}$ For every timelike covector field $\xi_{a} T^{a b} \xi_{a}$ is timelike and $T^{a b} \xi_{a} \xi_{b} \geq 0$ in any scale gauge ( $T$ not everywhere zero, here already clear).

23 Whether a scale invariant geodesic or a scale covariant geodesic of weight -1 , depends on the reparametrization.
} 
Geroch-Jang theorem are satisfied; thus $\gamma$ can be re-parametrized as a Levi-Civita geodesic in Riemann gauge $(\tilde{g}, 0)$. In $(\tilde{g}, 0)$ the Weyl geometric derivative $\nabla$ coincides with $\tilde{g} \nabla$ (the Levi-Civita derivation of $\tilde{g}$ ), thus $\tilde{\gamma}$ is a scale invariant geodesic of the Weyl structure (i.e., $\nabla_{\dot{\tilde{\gamma}}} \dot{\tilde{\gamma}}=0$ ). An appropriate re-parametrization gives it unit norm in any other gauge.

Dynamical interpretation If a scale co/in-variant theory of gravity is formulated in the framework of integrable Weyl geometry (IWG), the energy-momentum 2-form of matter $T_{a b}$ is of weight $-2 .^{24}$ Independent of which scale gauge expresses the observable quantities most directly_in Weyl geometric scalar tensor theory (WST) it is the Einstein gauge - a test body may be understood as the limit of small energymomentum tubes in this gauge. Then the conditions (i), (ii) are satisfied in this gauge, therefore in any one, and the theorem can be applied. Result: The geodesic principle holds in any reasonable dynamical theory of gravity in IWG.

\subsection{Short comment on gravitational light deflection}

According to a method used in [12, p. 288ff.] (following an approach outlined in [51]) the deflection angle $\alpha$ of the spatial wave vector of a small wave package travelling along null geodesics can be expressed, in a first order approximation in $h$, in terms of the spatial gradient $\vec{\nabla}$ of $\frac{1}{2}\left(-h_{00}+h_{j j}\right)$ for any $1 \leq j \leq 3$ (no summation over $j$ ). But here, like in other places of the literature, the special case of a pressure-less matter energy-momentum is assumed. A similar, in the result a bit more general, derivation of the deflection potential (including the case of moving masses) is given in [57, (4.19), p. 124]. Here the authors add an explanation of the result by using the Fermat principle. In the Fermat approach it becomes transparent that in our slightly more general constellation the deflection potential is given by $\frac{1}{2}\left(-h_{00}+h_{j j}\right)$. The authors use a conformally stationary spacetime with metric $d s^{2}=e^{2 U}\left(d t-w_{i} d x^{i}\right)^{2}-e^{2 U} d l^{2}$ (Eq. 3.35). The transition from (3.35) to the Fermat principle (3.39) $\delta \int_{\tilde{\gamma}}\left(w_{i} d x^{i}+e^{-2 U} d l\right.$ ) proceeds via specialization to null curves, $d s^{2}=0$, which leads to $d t=w_{i} d x^{i}+$ $e^{-2 U} d l$ (3.37). Generalizing (3.35) to $d s^{2}=e^{2 U}\left(d t-w_{i} d x^{i}\right)^{2}-e^{2 \tilde{U}} d l^{2}$ changes the null curve condition to (3.37') $d t=w_{i} d x^{i}+e^{-(U+\tilde{U})} d l^{2}$ and the Fermat principle to

$$
\delta \int_{\tilde{\gamma}}\left(w_{i} d x^{i}+e^{-(U+\tilde{U})} d l\right)
$$

(Similar at other places, e.g., [66, chap. IX, §3].) For a static metric (with $w_{i}=0$ ) the spatial projections of light rays are geodesics of a Riemannian metric with arc length $e^{-(U+\tilde{U})} d l$, different from the "physical" arc length $e^{-\tilde{U}} d l$. A similar remark is given for the more special situation in [57, p. 104].

${ }^{24}$ In a Lagrangian formulation the scale invariance of the matter Lagrangian $\mathfrak{L}_{m}=L_{m} \sqrt{|g|}$ demands/implies $w\left(L_{m}\right)=-4$. The variational derivative $T_{a b}=\frac{\delta L_{m}}{\delta g a b}$ increases the weight by 2 . 


\subsection{Comments on $[59,60]$}

In $[59,60]$ it has been claimed that a scalar field, nonminimally coupled to the Hilbert action and with the cubic kinetic term $L_{D \phi^{3}}$ (but without $L_{D^{2} \phi}$ ), can already bring new insight for the dark matter/modified gravity problem if is dealt with in framework of Weyl geometric gravity. Due to a flawed heuristic treatment of the weak field approximation, the energy momentum expression of the scalar field resulting from the variation of the non-minimal coupling (here Eq. 19) was interpreted as an effective contribution to the energy-momentum of the scalar field. But as we can see from (39) the energy-momentum expression (19) cancels with other terms of the Weyl geometric equation (most importantly the scale connection contribution to the Weyl geometric Einstein tensor). So this interpretation was wrong. On the other hand, some of the statements of the papers, regarding the emulation of the deep MOND kinematics and basic claims on the energy momentum of the scalar field, become correct in the present approach.

\section{References}

1. Almeida, T.S., Formiga, J.B., Pucheu, M.L., Romero, C.: From Brans-Dicke gravity to a geometrical scalar-tensor theory. Phys. Rev. D 89, 064047 (2014)

2. Bekenstein, J.: Relativistic gravitation theory for the modified Newtonian dynamics paradigm. Phys. Rev. D 70, 083509 (2004)

3. Bekenstein, J., Milgrom, M.: Does the missing mass problem signal the breakdown of Newtonian gravity? Astrophys. J. 286, 7-14 (1984)

4. Berezhiani, L., Famaey, B., Khoury, J.: Phenomenological consequences of superfluid dark matter with baryon-phonon coupling. arXiv:1711.05748 (2017)

5. Berezhiani, L., Khoury, J.: Theory of dark matter superfluidity. Phys. Rev. D 92, 103510 (2015)

6. Berezhiani, L., Khoury, J.: Dark matter superfluidity and galactic dynamics. Phys. Lett. B 753, 639-643 (2016)

7. Berezhiani, L., Khoury, J.: Emergent long-range interactions in Bose-Einstein condensates. Phys. Rev. D 99, 076003 (2019)

8. Bergmann, P.G.: Introduction to the Theory of Relativity. Englewood Cliffs, Prentice Hall. Reprint Dover, New York, 1976 (1942)

9. Blagojević, M.: Gravitation and Gauge Symmetries. Institute of Physics Publishing, Bristol (2002)

10. Calderbank, D., Pedersen, H.: Einstein-Weyl geometry. Adv. Math. 97, 74-109 (1998)

11. Capozziello, S., Faraoni, V.: Beyond Einstein Gravity. A Survey of Gravitational Theories for Cosmology and Astrophysics. Springer, Dordrecht (2011)

12. Carroll, S.: Spacetime and Geometry. Addison Wesley, San Francisco (2004)

13. Cheng, H.: Possible existence of Weyl's vector meson. Phys. Rev. Lett. 61, 2182-2184 (1988)

14. Clifton, T., Ferreira, P., Padilla, A., Skordis, C.: Modified gravity and cosmology. Phys. Rep. 513, 1-189 (2012)

15. Codello, A., D'Orodico, G., Pagani, C., Percacci, R.: The renormalization group and Weyl invariance. Class. Quantum Gravity 30, 115015 (2013)

16. Dahia, F., Gomez, A.T., Carlos, R.: On the embedding of space-time in five-dimensional Weyl spaces. J. Math. Phys. 49, 102501 (2008)

17. de Oliveira, H.P., Salim, J.M., Sautu, S.L.: Non-singular inflationary cosmologies in Weyl integrable spacetime. Class. Quantum Gravity 14(10), 2833-2843 (1997)

18. Dirac, P.A.M.: Long range forces and broken symmetries. Proc. R. Soc. Lond. A 333, $403-418$ (1973)

19. Drechsler, W., Tann, H.: Broken Weyl invariance and the origin of mass. Found. Phys. 29(7), 1023-1064 (1999)

20. Eddington, A.S.: The Mathematical Theory of Relativity, 2nd edn. University Press, Cambridge (1923) 
21. Famaey, B., McGaugh, S.: Modified Newtonian dynamics (MOND): observational phenomenology and relativistic extensions. Living Rev. Relativ. 15(10), 1-159 (2012)

22. Folland, G.B.: Weyl manifolds. J. Differ. Geom. 4, 145-153 (1970)

23. Frankel, T.: The Geometry of Physics. University Press, Cambridge (2004)

24. Fujii, Y., Maeda, K.-C.: The Scalar-Tensor Theory of Gravitation. University Press, Cambridge (2003)

25. Gauduchon, P.: La 1-forme de torsion d'une variété hermitienne compacte. Journal für die reine und angewandte Mathematik 469, 1-50 (1995)

26. Geroch, R., Jang, P.S.: Motion of a body in general relativity. J. Math. Phys. 16, 65-67 (1975)

27. Ghilencea, D.M.: Spontaneous breaking of Weyl quadratic gravity to Einstein action and Higgs potential. J. High Energy Phys. (2019). https://doi.org/10.1007/JHEP03(2019)049

28. Gilkey, P., Nikcevic, S., Udo, S.: Geometric realizations, curvature decompositions, and Weyl manifolds. J. Geom. Phys. 61, 270-275 (2011)

29. Higa, T.: Weyl manifolds and Einstein-Weyl manifolds. Commentarii Mathematici Sancti Pauli 42, 143-160 (1993)

30. Hossenfelder, S.: A covariant version of Verlinde's emergent gravity. Phys. Rev. D 95, 124018 (2017)

31. Hossenfelder, S., Mistele, T.: The redshift-dependence of radial acceleration: modified gravity versus particle dark matter. Int. J. Mod. Phys. D 27(14), 1847010 (2018)

32. Hossenfelder, S., Mistele, T.: The Milky Way's rotation curve with superfluid dark matter. arXiv:1803.08683 (2020)

33. Israelit, M.: The Weyl-Dirac Theory and Our Universe. Nova Science, New York (1999)

34. Jacobson, T., Mattingly, D.: Gravity with a dynamical preferred frame. Phys. Rev. D 64, 024028 (2001)

35. Joyce, A., Jain, B., Khoury, J., Trodden, M.: Beyond the cosmological standard model. Phys. Rep. 568, 1-98 (2017)

36. Lehmkuhl, D., Scholz, E.: The Einstein-Weyl correspondence and a geodesic theorem for a new type of spacetime (Forthcoming). Manuscript

37. Martens, N., Lehmkuhl, D.: Dark Matter = Modified Gravity? Scrutinising the spacetime-matter distinction through the modified gravity/dark matter lens. Manuscript (2019)

38. McGaugh, S.: The imprint of spiral arms on the galactic rotation curve. Astrophys. J. 885, 87 (2019)

39. McGaugh, S., Lelli, F., Schombert, J.M.: Radial acceleration relation in rotationally supported galaxies. Phys. Rev. Lett. 117(20), 201101 (2016)

40. Milgrom, M.: A modification of Newtonian dynamcis as a possible alternative to the hidden matter hypothesis. Astrophys. J. 270, 365-370 (1983)

41. Miritzis, J.: Isotropic cosmologies in Weyl geometry. Class. Quantum Gravity 21, 3043-3056 (2004)

42. Novello, M., Oliveira, L.A.R., Salim, J.M., Elbaz, E.: Geometrized instantons and the creation of the universe. Int. J. Mod. Phys. D 1, 641-677 (1993)

43. Ohanian, H.: Weyl gauge-vector and complex dilaton scalar for conformal symmetry and its breaking. Gen. Relativ. Gravit. (2016). https://doi.org/10.1007/s10714-016-2023-8

44. Olling, R.P., Merrifield, M.R.: Luminous and dark matter in the Milky Way. Mon. Not. R. Astron. Soc. 326, 164 (2001)

45. Oloff, R.: Goemtrie der Raumzeit. Vieweg, Brauschweig/Wiesbaden (1999)

46. Omote, M.: Scale transformations of the second kind and the Weyl space-time. Lettere al Nuovo Cimento 2(2), 58-60 (1971)

47. O’Raifeartaigh, L.: The Dawning of Gauge Theory. University Press, Princeton (1997)

48. Pauli, W.: Relativitätstheorie. Neu herausgegeben und kommentiert von Domenico Giulini. Berlin: Springer (1921/2000)

49. Perlick, V.: Zur Kinematik Weylscher Raum-Zeit-Modelle. Dissertationsschrift. Berlin: Fachbereich Physik, TU Berlin (1989)

50. Poulis, F.P., Salim, J.M.: Weyl geometry as a characterization of space-time. Int. J. Mod. Phys. Conf. Ser. V 3, 87-97 (2011)

51. Pyne, T., Birkinshaw, M.: Beyond the thin lens approximation. Astrophys. J. 458, 46 (1996)

52. Quiros, I.: Scale invariant theory of gravity and the standard model of particles. arXiv:1401.2643 (2014)

53. Reiprich, T.: Cosmological Implications and Physical Properties of an X-Ray Flux-Limited Sample of Galaxy Clusters. Dissertation University Munich (2001)

54. Riess, A., Casertano, S., Yuan, W., Macri, L., Scolnic, D.: Large magellanic cloud cepheid standards provide a $1 \%$ foundation for the determination of the Hubble constant and stronger evidence for physics beyond $\Lambda C D M$. Astgrophys. J. arXiv:1903.07603 (2019, accepted) 
55. Romero, C., Fonseca-Neto, J.B., Pucheu, M.L.: General relativity and Weyl frames. Int. J. Mod. Phys. A 26(22), 3721-3729 (2011)

56. Sanders, R.H.: The Dark Matter Problem. A Historical Perspective. University Press, Cambridge (2010)

57. Schneider, P., Ehlers, J., Falco, E.E.: Gravitational Lenses. Springer, Berlin (1992)

58. Scholz, E.: Weyl geometric gravity and electroweak symmetry breaking. Ann. Phys. 523, 507-530 (2011)

59. Scholz, E.: Clusters of galaxies in a Weyl geometric approach to gravity. J. Gravity 9706704 (2016). arXiv:1506.09138. Corrigendum in [61]

60. Scholz, E.: MOND-like acceleration in integrable Weyl geometric gravity. Found. Phys. 46, 176-208 (2016)

61. Scholz, E.: Corrigendum to "Clusters of Galaxies in a Weyl Geometric Approach to Gravity". J. Gravity (2017). https://doi.org/10.1155/2017/9151485

62. Scholz, E.: The unexpected resurgence of Weyl gometry in late 20-th century physics. In: Walter, S., Rowe, D., Sauer, T. (eds.) Beyond Einstein. Perspectives on Geometry, Gravitation and Cosmology, Vol. 13 of Einstein Studies, pp. 261-360. Springer, Berlin (2018)

63. Shaposhnikov, M., Zenhäusern, D.: Quantum scale invariance, cosmological constant and hierarchy problem. Phys. Lett. B 671, 162-166 (2009)

64. Skordis, C.: Generalizing tensor-vector-scalar cosmology. Phys. Rev. D 77, 123502 (2008)

65. Smolin, L.: Towards a theory of spacetime structure at very short distances. Nucl. Phys. B 160, 253-268 (1979)

66. Synge, J.L.: Relativity: The General Theory. North Holland, Amsterdam (1960)

67. Tann, H.: Einbettung der Quantentheorie eines Skalarfeldes in eine Weyl Geometrie-Weyl Symmetrie und ihre Brechung. Utz, München (1998)

68. Tonnelat, M.-A.: Les Théories unitaires de l'électromagnétisme et de la gravitation. Gauthier-Villars, Paris (1965)

69. Utiyama, R.: On Weyl's gauge field. Prog. Theor. Phys. 50, 2028-2090 (1973)

70. Weinberg, S.: Gravitation and Cosmology. Wiley, New York (1972)

71. Weyl, H.: Gravitation und Elektrizität. Sitzungsberichte der Königlich Preußischen Akademie der Wissenschaften zu Berlin, pp. 465-480. In [74, II, 29-42] [31], English in [47, 24-37] (1918)

72. Weyl, H.: Reine Infinitesimalgeometrie. Mathematische Zeitschrift 2:384-411. In [74, II, 1-28] [30] (1918)

73. Weyl, H.: 1922. Space-Time-Matter. Translated from the 4th German edition by H. Brose. Methuen, London. Reprint Dover, New York (1952)

74. Weyl, H.: Gesammelte Abhandlungen, vol. 4. Springer, Berlin (1968)

75. Will, C.: The confrontation between general relativity and experiment. Living Rev. Relativ. 4, 1-97 (2001)

76. Yuan, F.-F., Huang, Y.-C.: A modified variational principle for gravity in Weyl geometry. Class. Quantum Gravity 30(19), 195008 (2013)

77. Zhang, Y.-Y., Laganá, T.F., Pierini, D., Puchwein, E., Schneider, P., Reiprich, T.H.: Star-formation efficiency and metal enrichment of the intracluster medium in local massive clusters of galaxies. Astron. Astrophys. 535(A78), 11 (2011)

78. Zhang, Y.-Y., Laganá, T.F., Pierini, D., Puchwein, E., Schneider, P., Reiprich, T.H.: Corrigendum to star-formation efficiency and metal enrichment of the intracluster medium in local massive clusters of galaxies. Astron. Astrophys. 544(C3), 1 (2012)

Publisher's Note Springer Nature remains neutral with regard to jurisdictional claims in published maps and institutional affiliations. 